\title{
Opportunistic control over shared wireless channels
}

\author{
Konstantinos Gatsis, Miroslav Pajic, Alejandro Ribeiro, and George J. Pappas
}

\begin{abstract}
We consider a wireless control architecture with multiple control loops and a shared wireless medium. A scheduler observes the random channel conditions that each control system experiences over the shared medium and opportunistically selects systems to transmit at a set of non-overlapping frequencies. The transmit power of each system also adapts to channel conditions and determines the probability of successfully transmitting and closing the loop. We formulate the problem of designing optimal channel-aware scheduling and power allocation mechanisms that minimize the total power consumption while meeting control performance requirements for all systems. In particular it is required that for each control system a given Lyapunov function decreases at a specified rate in expectation over the random channel conditions. We develop an offline algorithm to find the optimal communication design, as well as an online protocol which selects scheduling and power variables based on a random observed channel sequence and converges almost surely to the optimal operating point. We illustrate in simulations the power savings of our approach compared to other non-channel-aware schemes.
\end{abstract}

\section{INTRODUCTION}

Modern wireless control environments in e.g., smart buildings or industrial applications, include numerous wireless sensor and actuators communicating with controllers. The need for efficiently sharing the available wireless medium between these devices becomes apparent. Scheduling access to the shared medium helps eliminate interferences between transmissions, but the rate at which a control system accesses the medium affects closed loop performance. This necessitates the development of wireless communication and resource management mechanisms that are control-aware. Moreover, since wireless devices in such applications are often batteryoperated, these mechanisms are desired to be energy-efficient.

Scheduling in wired or wireless networked control systems has received a lot of attention in the past. Scheduling mechanisms usually examined are either static or dynamic. Typical examples of the first type are periodically protocols where the wireless devices transmit in a predefined repeating order, e.g., round-robin. Stability conditions under such scheduling protocols can be examined by converting the system in some form of a switching system, usually including other network phenomena such as delays, uncertain communication times, or packet drops - see, e.g., [2]-[5]. The problem of designing static schedules suitable for control applications has also been addressed. Periodic sequences leading to stability [6], controllability and observability [7], or minimizing linear quadratic

This work was supported in part by NSF CNS-0931239, and by TerraSwarm, one of six centers of STARnet, a Semiconductor Research Corporation program sponsored by MARCO and DARPA. The authors are with the Department of Electrical and Systems Engineering, University of Pennsylvania, 200 South 33rd Street, Philadelphia, PA 19104. Email: \{kgatsis, pajic, aribeiro, pappasg\}@seas.upenn.edu. Part of the results in this paper appeared at ICCPS'14 [1]. objectives [8] have been proposed. Deriving otherwise optimal scheduling sequences is recognized as a hard combinatorial problem [9], [10].

Dynamic schedulers on the other hand do not rely on a predefined sequence but decide access to the communication medium at each step, for example by dynamically assigning priorities to the competing tasks. Priorities commonly depend on the current plant/control system states, i.e., informally speaking, the subsystem with the largest state discrepancy is scheduled to communicate. Examples of such dynamic schedulers can be found in [4], [11]-[13]. Recent efforts have also focused on scheduling event-based controllers [14], [15]. Another approach, motivated by the problem of scheduling control tasks sharing a computation (CPU) rather than a communication resource, is to abstract control performance requirements in the time/frequency domain. Knowing for example how often a task needs access to the resource, to communicate and close the loop in our case, static/offline and dynamic/online schedules meeting the desired requirements can be obtained using algorithms from real-time scheduling theory [16], [17].

However, in the case of multiple control tasks sharing a wireless communication medium the existing scheduling mechanisms in the control literature do not explicitly model or account for the wireless physical layer aspects of the problem. In particular, time-varying channel conditions cause large unpredictable variations in wireless channel transferences, referred to as fading [18, Ch. 3,4]. The problem of designing wireless communication networks to counteract such channel variability and maximize the utility to the users has received considerable attention [19]-[21]. The aim in these works is to allocate the available communication resources, e.g., medium access, power resources, channel capacity, by opportunistically adapting to the randomly varying channel conditions.

In this paper we propose a channel-aware approach for scheduling independent control tasks sharing a wireless communication medium (Fig. 1). The channel conditions on the medium not only change randomly over time, but also differ among the control systems at a given time step. We consider a scheduler that selects at most one control system to transmit over each of a number of available frequencies at each time step. Such a channel-aware mechanism can opportunistically exploit channel information to, e.g., grant channel access to control loops experiencing favorable channel conditions, or equivalently avoid closing the loop under adverse conditions. Similar to our previous work for single-loop systems in [22], [23] we allow for the selection of transmit power when a system is scheduled. Transmit power and channel fading determine the probability of successful message delivery at the receiver.

Our opportunistic scheduling and power policies are de- 
signed to serve a set of predesigned control tasks over the shared wireless medium. To enable a control-aware communication design, a suitable abstraction of the control system dynamics and performance requirements is required. In this paper each control system is abstracted by some given Lyapunov function and control performance is specified as a desired decrease rate for this Lyapunov function (Section II). Since scheduling and power allocation in our design depend on random wireless channel conditions our control performance guarantees are expressed in a stochastic sense. Our design is required to ensure that all Lyapunov functions decrease at the specified rates at every time step in expectation over the channel conditions. Note that, in contrast to this stochastic requirement, at most one loop closes at any time step deterministically. As a result we express control performance requirements in a static single-time-step framework unlike, e.g., timing/frequency abstractions [16] or periodic sequences [7] which would be hard to analyze under random wireless communication. We also point out that previous works often construct Lyapunov functions to prove stability under proposed schedulers [4], [6], [14], while in contrast here we employ Lyapunov functions as a control performance specification to enable design of our scheduler.

We formulate the problem of optimal channel-aware scheduling and power allocation that minimize the expected total power consumption subject to the expected Lyapunov decrease rate constraints (Section II-A). In Section III we develop an offline algorithm to solve the problem in the dual domain and characterize the form of the optimal solution. The optimal power allocation is decentralized among users and frequencies, and the optimal scheduler opportunistically assigns control systems to frequencies depending on channel conditions. Moreover, in Section IV we develop an online communication algorithm which, unlike the offline one, does not require knowledge of the channel distribution but utilizes only a channel sequence observed during execution. The online algorithm bears an intuitive pricing interpretation (Section IV-A), and we establish that if scheduling and power allocation are selected this way, the desired Lyapunov performance constraints are met in the limit in a strong sense (almost surely). Finally, simulations in Section V illustrate the opportunistic nature of our channel-aware approach as well as the reduction in power consumption (at a magnitude of $80 \%$ in examples) compared to non-channel-aware mechanisms. We conclude with a discussion and future research directions in Section VI.

Notation: We denote the real $m$-dimensional non-negative orthant with $\mathbb{R}_{+}^{m}$, and the comparison with respect to the orthant (i.e., element-wise) with $\geq$, i.e., $x \geq y$ if and only if $x-y \in \mathbb{R}_{+}^{m}$. The cone of $n \times n$ real symmetric positive semidefinite matrices is denoted by $S_{+}^{n}$, and the comparison with respect to this cone with $\succeq$. The set of $n \times n$ real symmetric positive definite matrices is denoted by $S_{++}^{n}$.

\section{PRoblem DESCRIPTION}

Consider the wireless control architecture of Fig. 1 consisting of $m$ independent networked control systems. Each control

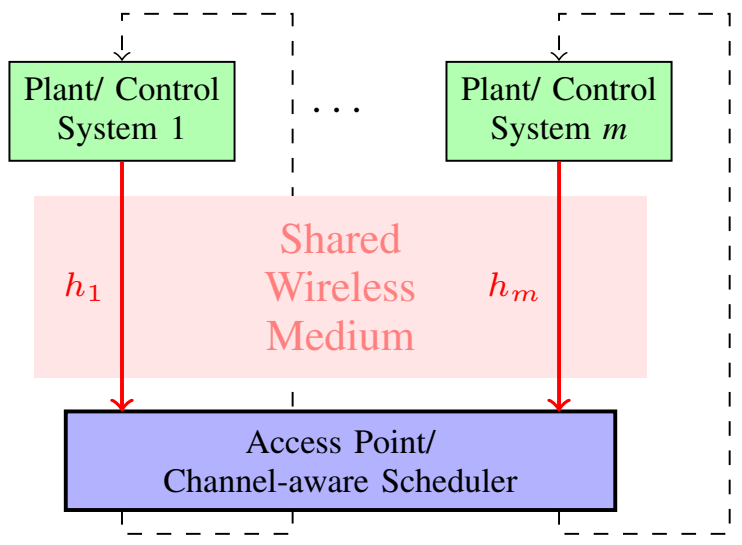

Fig. 1. Architecture for opportunistic scheduling of control tasks over a shared wireless medium. Independent control systems close the loop by transmitting over the shared wireless medium to a common receiver/access point. For simplicity the case of a single transmission frequency is shown, where each control system $i$ experiences random channel conditions $h_{i}$. A centralized scheduler located at the access point observes all channel states and opportunistically decides which system is scheduled to transmit and close the loop.

loop $i(i=1,2, \ldots, m)$ includes a wireless transmitter communicating to a common receiver/access point. For example this can be a wireless sensor transmitting plant measurements to a common access point responsible for computing the control inputs to the systems. A centralized scheduler, implemented at the access point, decides which control system is given access to the shared wireless channel.

We denote the state of system $i$ at each time $k$ by $x_{i, k} \in$ $\mathbb{R}^{n_{i}}$. We assume that different descriptions for the evolution of each system $i$ from $x_{i, k}$ to $x_{i, k+1}$ are given depending on whether a transmission occurs at time $k$ or not. Let us indicate with $\gamma_{i, k} \in\{0,1\}$ the event that a successful transmission occurs at time $k$ for the subsystem $i$. Then we describe the system evolution by a switched linear time invariant model,

$$
x_{i, k+1}=\left\{\begin{array}{ll}
A_{c, i} x_{i, k}+w_{i, k}, & \text { if } \gamma_{i, k}=1 \\
A_{o, i} x_{i, k}+w_{i, k}, & \text { if } \gamma_{i, k}=0
\end{array} .\right.
$$

At a successful transmission the system dynamics are described by the matrix $A_{c, i} \in \mathbb{R}^{n_{i} \times n_{i}}$, where 'c' stands for closed-loop, and otherwise by $A_{o, i} \in \mathbb{R}^{n_{i} \times n_{i}}$, where 'o' stands for open-loop. We assume that $A_{c, i}$ is asymptotically stable, implying that if system $i$ were to transmit at each slot its respective state evolution is stable. The open loop matrix $A_{o, i}$ may be unstable. The additive terms $w_{i, k}$ model an independent identically distributed (i.i.d.) noise process with mean zero and covariance $W_{i} \succeq 0$. Note that the noise terms are modeled as independent across time $k$ for each plant $i$ and also independent across plants $i$. Furthermore, it is worth noting that closed-loop dynamics for all of the $m$ systems are fixed, meaning that adequate controllers have been already designed. In this paper we focus on designing the wireless communication aspects of the control system. The above networked control system description (1) can model various control operations, as shown in the following examples.

Example 1. Suppose each closed loop $i$ consists of a linear 
plant of the form

$$
x_{i, k+1}=A_{i} x_{i, k}+B_{i} u_{i, k}+w_{i, k},
$$

and a wireless sensor transmitting the plant state measurement $x_{i, k}$ to a controller/actuator which provides input $u_{i, k}$. Let then the controller apply a linear feedback $u_{i, k}=K_{i} x_{i, k}$ when a measurement is received $\left(\gamma_{i, k}=1\right)$, otherwise apply for simplicity $u_{i, k}=0$ when no measurement is received $\left(\gamma_{i, k}=\right.$ $0)$. The resulting closed loop system can be written as

$$
x_{i, k+1}=\left\{\begin{array}{ll}
\left(A_{i}+B_{i} K_{i}\right) x_{i, k}+w_{i, k}, & \text { if } \gamma_{i, k}=1 \\
A_{i} x_{i, k}+w_{i, k}, & \text { if } \gamma_{i, k}=0
\end{array} .\right.
$$

which is of the form (1) with $A_{c, i}=A_{i}+B_{i} K_{i}$ and $A_{o, i}=A_{i}$.

Example 2. As a more general example consider again the plants in (2) and wireless sensors, one for each plant, measuring system outputs of the form

$$
y_{i, k}=C_{i} x_{i, k}+v_{i, k},
$$

where $v_{i, k}$ is some i.i.d. measurement noise with zero mean and finite covariance. A dynamic control law based on this plant output and adapted to the packet drops updates a local controller state according to

$$
z_{i, k+1}=F_{i} z_{i, k}+\gamma_{i, k}\left(F_{c, i} z_{i, k}+G_{i} y_{i, k}\right),
$$

i.e., corrects appropriately the local state whenever a measurement is received. For example $z_{i, k}$ may represent a local estimate of the plant state [2]. The control input applied by the controller can similarly be modeled as

$$
u_{i, k}=K_{i} z_{i, k}+\gamma_{i, k}\left(K_{c, i} z_{i, k}+L_{i} y_{i, k}\right),
$$

The overall closed loop system is obtained by joining plant and controller states into

$$
\begin{gathered}
{\left[\begin{array}{c}
x_{i, k+1} \\
z_{i, k+1}
\end{array}\right]=\left[\begin{array}{cc}
A_{i} & B_{i} K_{i} \\
0 & F_{i}
\end{array}\right]\left[\begin{array}{l}
x_{i, k} \\
z_{i, k}
\end{array}\right]+\left[\begin{array}{c}
I \\
0
\end{array}\right] w_{i, k}+} \\
\gamma_{i, k}\left[\begin{array}{cc}
B_{i} L_{i} C_{i} & B_{i} K_{c, i} \\
G_{i} C_{i} & F_{c, i}
\end{array}\right]\left[\begin{array}{l}
x_{i, k} \\
z_{i, k}
\end{array}\right]+\gamma_{i, k}\left[\begin{array}{c}
B_{i} L_{i} C_{i} \\
G_{i}
\end{array}\right] v_{i, k},
\end{gathered}
$$

which is again of the form (1).

Let us now describe the wireless communication system and model how it determines the packet transmission successes, i.e., the indicators $\gamma_{i, k}$. Suppose there are $f$ different frequencies that each system may use to communicate to the access point and let the wireless channel conditions for a system $i$ and frequency $j$ at time slot $k$ be denoted as $h_{i j, k}$. Channel conditions $h_{i j, k}$ refer to the channel fading coefficient that system $i$ experiences if it transmits at time slot $k$ over frequency $j$. Due to propagation effects the channel fading $h_{i j, k}$ changes unpredictably [18, Ch. 3] and takes values in a subset $\mathcal{H} \subseteq \mathbb{R}_{+}$of the positive reals. We adopt a block fading model [18, Ch. 4] whereby channel states $\left\{h_{i j, k}, 1 \leq i \leq m, 1 \leq j \leq f\right\}$ are modeled as constant during each transmission slot $k$, but independent and identically distributed across different time slots $k$ according to some joint distribution $\phi$ on $\mathcal{H}^{m \times f}$. They are also independent of the plant process noise $w_{i, k}$. We assume the channel states

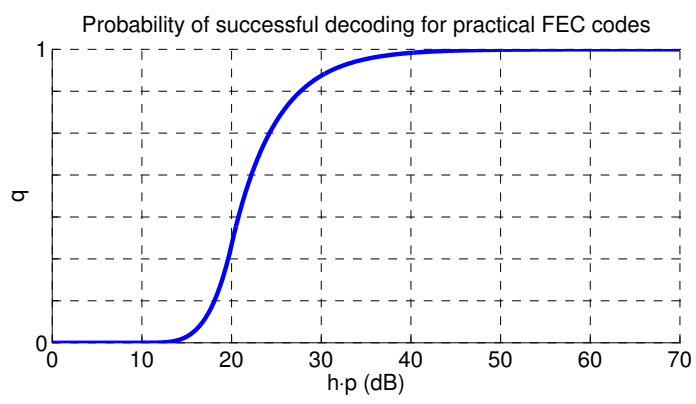

Fig. 2. Complementary error function for practical FEC codes. The probability of successful decoding $q$ for a FEC code is a sigmoid function of the received $\mathrm{SNR} \sim h p$.

are available to the access point before transmission - see Remark 1 for a practical implementation. We also make the following technical assumption on their joint distribution to exclude the possibility of channel states becoming degenerate random variables.

Assumption 1. The joint distribution $\phi$ of channel states $\left\{h_{i j, k}, 1 \leq i \leq m, 1 \leq j \leq f\right\}$ has a probability density function on $\mathcal{H}^{m \times f}$.

If system $i$ transmits at time $k$ over frequency $j$ it selects a transmit power level $p_{i j, k}$ taking values in $\left[0, p_{\max }\right]$. Then channel fading and transmit power affect the probability of successful decoding of the transmitted packet at the receiver. In particular given the forward error-correcting code (FEC) in use, the probability $q$ that a packet is successfully decoded is a function of the received signal-to-noise ratio (SNR). The SNR is proportional to the received power level expressed by the product $h \cdot p$ of channel fading and the allocated transmit power. Overall we express the probability of success by a given relationship of the form $q\left(h_{i j, k} \cdot p_{i j, k}\right)$ - for more details on this model, the reader is referred to [22]. An illustration of this relationship is given in Fig. 2. The following technical assumption on the form of the function $q(h p)$ will be helpful in the subsequent sections.

Assumption 2. The function $q($.$) as a function of the product$ $r=h p$ for $r \geq 0$ satisfies:

(a) $q(0)=0$,

(b) $q(r)$ is continuous, and strictly increasing when $q(r)>0$, i.e., for $r^{\prime}>r$ it holds that $q\left(r^{\prime}\right)>q(r)>0$,

(c) for any $\mu \geq 0$ and for almost all values $h \in \mathcal{H}$ the set $\operatorname{argmin}_{0 \leq p \leq p_{\max }} p-\mu q(h p)$ is a singleton.

Parts (a) and (b) of this assumption state that the probability of successful decoding $q(h p)$ is zero when the received power level $h p$ is small, and it becomes positive $q(h p)>0$ and strictly increasing for larger values of $h p$. These properties are verified for cases of practical interest as shown in Fig. 2. Part (c) is more stringent but not restrictive in practice. The function $q(h p)$ typically has a sigmoid form with exponential tails as shown in Fig. 2. This verifies that the power minimizer in (c) is unique for almost all channel gains $h$, and is either equal to zero or belongs to the strictly concave exponential tail. We also note that the minimizer set in (c) exists by the 
continuity assumption in (b). These properties are assumed for technical reasons that will become clear later (see the discussion before Theorem 1).

Apart from packet drops due to low received SNR, packet collisions may occur if more than one of the control systems transmit at a given time slot on the same frequency over the shared wireless medium. For this reason we are interested in designing a mechanism to select which system accesses each of the available frequencies at the channel, i.e., which system is scheduled to transmit. We denote with $\alpha_{i j, k}=1$ the decision to schedule system $i$ on frequency $j$ at time $k$, and $\alpha_{i j, k}=0$ otherwise. To avoid packet collisions we let at most one system transmit on each frequency $j$, that is $\sum_{i=1}^{m} \alpha_{i j, k} \leq 1$. We allow each system $i$ to transmit on at most one frequency, that is $\sum_{j=1}^{f} \alpha_{i j, k} \leq 1$. Mathematically we may denote then the set $\Delta_{m, f}$ of all feasible scheduling decisions $\alpha_{i j, k}$ at each time $k$ as

$$
\Delta_{m, f}=\left\{\alpha \in\{0,1\}^{m \times f}: \begin{array}{l}
\sum_{i=1}^{m} \alpha_{i j} \leq 1,1 \leq j \leq f \\
\sum_{j=1}^{f} \alpha_{i j} \leq 1,1 \leq i \leq m
\end{array}\right\}
$$

For compactness we group channel states, scheduling decisions, and power allocations of the overall communication system at time $k$ into matrices $h_{k} \in \mathcal{H}^{m \times f}, \alpha_{k} \in \Delta_{m, f}$, and $p_{k} \in\left[0, p_{\max }\right]^{m \times f}$ respectively. We can then model the transmission event $\gamma_{i, k}$ of system $i$ at time $k$ given scheduling variables, power allocation, and channel state, as a Bernoulli random variable with success probability

$$
\mathbb{P}\left[\gamma_{i, k}=1 \mid h_{k}, \alpha_{k}, p_{k}\right]=\sum_{j=1}^{f} \alpha_{i j, k} q\left(h_{i j, k}, p_{i j, k}\right)
$$

This expression states that the probability of a message for system $i$ being successfully received equals the probability that the message is correctly decoded if system $i$ is scheduled to transmit on any of the $f$ available frequencies. Note that, by design of the scheduling variables, system $i$ uses at most one frequency, and we make the implicit assumption that no interferences arise from transmissions on different frequencies.

Our goal is to design the communication variables of the shared wireless control system, i.e., the scheduling and power allocation. Since the randomly varying channel affects the communication process, we are interested in selecting scheduling and power variables that adapt to channel states $h_{k}$ in order to counteract these effects. Overall we express the scheduling and power decisions $\alpha_{k}, p_{k}$ respectively as mappings of the form

$$
\begin{aligned}
& \mathcal{A}=\left\{\alpha: \mathcal{H}^{m \times f} \rightarrow \Delta_{m, f}\right\}, \\
& \mathcal{P}=\left\{p: \mathcal{H}^{m \times f} \rightarrow\left[0, p_{\text {max }}\right]^{m \times f}\right\},
\end{aligned}
$$

so that $\alpha_{k}=\alpha\left(h_{k}\right), p_{k}=p\left(h_{k}\right)$. Since channel states $h_{k}$ are i.i.d. over time $k$ these mappings do not need to change over time. Substituting the scheduling and power allocation mappings $\alpha(),. p($.$) in our communication model described by$ (9) the probability of successful transmission for each system $i$ at any given slot $k$ becomes

$$
\begin{aligned}
\mathbb{P}\left(\gamma_{i, k}=1\right) & =\mathbb{E}_{h_{k}}\left\{\mathbb{P}\left[\gamma_{i, k}=1 \mid h_{k}, \alpha\left(h_{k}\right), p\left(h_{k}\right)\right]\right\} \\
& =\mathbb{E}_{h} \sum_{j=1}^{f} \alpha_{i j}(h) q\left(h_{i j}, p_{i j}(h)\right) .
\end{aligned}
$$

Here the expectation is with respect to the joint distribution $\phi$ of the channel realization $h_{k}$ which we assumed to be identical for any time $k$, hence we drop the index $k$. Note also that the communication process modeled by the sequence $\left\{\gamma_{i, k}, 1 \leq i \leq m, k \geq 0\right\}$ depends only on variables related to the wireless communication counterpart of the overall system, and is in particular independent of the system evolutions $\left\{x_{i, k}, 1 \leq i \leq m, k \geq 0\right\}$.

Our primary goal in designing the communication variables of the system is to guarantee a level of closed loop control performance for each subsystem. To formalize the problem description we consider Lyapunov-like performance requirements for the control systems. In particular suppose that for each system $i$ a quadratic Lyapunov function of the form

$$
V_{i}\left(x_{i}\right)=x_{i}^{T} P_{i} x_{i}, x_{i} \in \mathbb{R}^{n_{i}},
$$

with positive definite matrix $P_{i} \in S_{++}^{n_{i}}$ is given. A Lyapunovlike requirement then states that these functions should decrease at given rates $\rho_{i}<1$ during the evolution of each subsystem $i$. This evolution however is random because of the stochastic nature of the wireless communication/control system, i.e., due to process noise, random channel states, randomized channel access, and packet drops. To take these effects into account we require that for all systems $i$ at time $k$ the Lyapunov functions at the next time step decrease at the desired rates $\rho_{i}<1$ in expectation, that is

$$
\mathbb{E}\left[V_{i}\left(x_{i, k+1}\right) \mid x_{i, k}\right] \leq \rho_{i} V_{i}\left(x_{i, k}\right)+\operatorname{Tr}\left(P_{i} W_{i}\right)
$$

for any possible value of the current plant states $x_{i, k} \in \mathbb{R}^{n_{i}}$. The expectation over the next system state $x_{i, k+1}$ on the left hand side accounts via (1) for the randomness introduced by the process noise $w_{i, k}$ as well as the transmission success $\gamma_{i, k}$. The latter is expressed in (11) and depends on the observed channel state $h_{k}$ as well as the communication decisions $\alpha_{k}, p_{k}$. The noise $w_{i, k}$ by (1) appears additively in the next plant state independently of the current transmission success and plant state. Since $V_{i}($.$) is quadratic and the$ noise has a covariance $W_{i}$, we conclude that that the term $\mathbb{E}\left[V_{i}\left(x_{i, k+1}\right) \mid x_{i, k}\right]$ includes a persistent noise perturbation equal to $\operatorname{Tr}\left(P_{i} W_{i}\right)$. That is the reason the constant $\operatorname{Tr}\left(P_{i} W_{i}\right)$ is artificially added at the right hand side of condition (13). The intuition behind condition (13) is as follows. If (13) holds for each time step $k=0, \ldots, N$, then by taking the expectation at both sides and by iterating backwards in time we find that

$$
\begin{aligned}
\mathbb{E} V_{i}\left(x_{i, N}\right) & \leq \rho_{i} \mathbb{E} V_{i}\left(x_{i, N-1}\right)+\operatorname{Tr}\left(P_{i} W_{i}\right) \\
& \leq \ldots \leq \rho_{i}^{N} \mathbb{E} V_{i}\left(x_{i, 0}\right)+\sum_{k=0}^{N-1} \rho_{i}^{k} \operatorname{Tr}\left(P_{i} W_{i}\right)
\end{aligned}
$$

Hence, system states have second moments that decay exponentially with rate $\rho_{i}$ with respect to initial states, and in the 
limit remain bounded by $\operatorname{Tr}\left(P_{i} W_{i}\right) /\left(1-\rho_{i}\right)$, since the sum in (14) converges due to $\rho_{i}<1$.

On the other hand, apart from control performance requirements an efficient communication design should make an efficient use of the available power resources at the devices. The induced overall expected power consumption on each slot $k$ is given by

$$
\mathbb{E}_{h_{k}} \sum_{i=1}^{m} \sum_{j=1}^{f} \alpha_{i j, k}\left(h_{k}\right) p_{i j, k}\left(h_{k}\right),
$$

summing up the transmit power of each system $i$ and frequency $j$ if the system is scheduled to transmit. The expectation here is with respect to the joint distribution $\phi$ of channels $h_{k}$. We design scheduling and power allocation (cf. (10)) that are control-performance aware (cf. (13)) and also energy-efficient (cf. (15)) through a stochastic optimization framework that we present next.

Remark 1. The centralized scheduler of the multiple access channel architecture in Fig. 1 requires channel state information. The channel conditions for each system $i$ can be measured at the access point at the beginning of each time slot by short pilot signals sent from the wireless transmitters of all systems to the access point. Depending on the measured channel states the access point decides which plant is scheduled to close the loop during the time slot. We note that perfect state information is not required, as $h$ can denote in practice an estimate of the channel state - see also [22]. We also point out that even though the pilot signals for the channel estimation incur some power consumption, we assume that practically this is much lower than the power necessary for transmitting the packets of the control systems, especially for large packet lengths (e.g. long headers). Hence the power for channel estimation is not included in our objective in (15).

\section{A. Scheduling and power allocation as stochastic optimization}

We formulate the problem of designing scheduling and power allocation in an optimization framework as follows.

Problem 1 (Optimal Scheduling and Power Allocation Design). Consider a shared wireless control architecture with $f$ frequencies and $m$ systems of the form (1), quadratic Lyapunov performance requirements by (13), channel states $h_{k} \in \mathcal{H}^{m \times f}$ i.i.d. with distribution $\phi$, and communication modeled by (9). The design of optimal scheduling and power allocation as functions of the current channel states $\alpha_{k}=$ $\alpha\left(h_{k}\right)$ and $p_{k}=p\left(h_{k}\right)$ respectively is posed as

$$
\begin{array}{ll}
\underset{\alpha, p \in(\mathcal{A}, \mathcal{P})}{\operatorname{minimize}} & \mathbb{E}_{h_{k}} \sum_{i=1}^{m} \sum_{j=1}^{f} \alpha_{i j, k}\left(h_{k}\right) p_{i j, k}\left(h_{k}\right) \\
\text { subject to } & \mathbb{E}\left[V_{i}\left(x_{i, k+1}\right) \mid x_{i, k}\right] \leq \rho_{i} V_{i}\left(x_{i, k}\right)+\operatorname{Tr}\left(P_{i} W_{i}\right) \\
& \text { for all } x_{i, k} \in \mathbb{R}^{n_{i}}, i=1, \ldots, m .
\end{array}
$$

In other words, at each time step we seek to minimize the total expected power consumption (15) of the design while satisfying the Lyapunov requirements (13) for all systems $i$ and for any value of the current plant states $x_{i, k} \in \mathbb{R}^{n_{i}}$, since scheduling and power allocation adapt to channel states but are independent of the plant states. To make explicit how the functions $\alpha(),. p($.$) appear in the constraints of the problem,$ i.e., the Lyapunov requirements, observe that by (1) we have that

$$
\begin{aligned}
& \mathbb{E}\left[V_{i}\left(x_{i, k+1}\right) \mid x_{i, k}\right]=\mathbb{P}\left(\gamma_{i, k}=1\right) x_{i, k}^{T} A_{c, i}^{T} P_{i} A_{c, i} x_{i, k} \\
& +\mathbb{P}\left(\gamma_{i, k}=0\right) x_{i, k}^{T} A_{o, i}^{T} P_{i} A_{o, i} x_{i, k}+\operatorname{Tr}\left(P_{i} W_{i}\right),
\end{aligned}
$$

where we used the fact that the random variable $\gamma_{i, k}$ is independent of the system state $x_{i, k}$ as it depends only on the communication variables (cf. (9)-(11)). Plugging (17) at the left hand side of the constraints in (16) we get for $x_{i, k} \neq 0$

$$
\mathbb{P}\left(\gamma_{i, k}=1\right) \geq \frac{x_{i, k}^{T}\left(A_{o, i}^{T} P_{i} A_{o, i}-\rho_{i} P_{i}\right) x_{i, k}}{x_{i, k}^{T}\left(A_{o, i}^{T} P_{i} A_{o, i}-A_{c, i}^{T} P_{i} A_{c, i}\right) x_{i, k}} .
$$

The decision variables $\alpha(),. p($.$) determine \mathbb{P}\left(\gamma_{i}=1\right)$ at the left hand side of this constraint by (11). Since condition (18) needs to hold at any value of $x_{i, k} \in \mathbb{R}^{n_{i}}$, we can rewrite it as $\mathbb{P}\left(\gamma_{i, k}=1\right) \geq c_{i}$ where

$$
c_{i}=\sup _{y \in \mathbb{R}^{n_{i}, y \neq 0}} \frac{y^{T}\left(A_{o, i}^{T} P_{i} A_{o, i}-\rho_{i} P_{i}\right) y}{y^{T}\left(A_{o, i}^{T} P_{i} A_{o, i}-A_{c, i}^{T} P_{i} A_{c, i}\right) y} .
$$

Computing $c_{i}$ is a simple semidefinite programming problem which can be easily solved using available convex optimization software. The value $c_{i}$ represents the minimum probability of transmission for each system $i$ that guarantees the desired Lyapunov decay rate $\rho_{i}-$ see also Remark 2. It can alternatively be thought of as a minimum required utilization factor of the shared wireless channel, analogously to a utilization of a shared CPU in, e.g., [17]. Intuitively, large value of $c_{i}$ implies that system $i$ requires more resources, i.e., more frequent channel access and possibly higher power expenditures.

Summarizing, the Lyapunov constraints in optimization (16) can be simplified by solving the auxiliary problems (19) for each control loop $i$, so that the optimization (16) can be equivalently written as

$$
\begin{array}{ll}
\underset{\alpha, p \in(\mathcal{A}, \mathcal{P})}{\operatorname{minimize}} & \mathbb{E}_{h} \sum_{i=1}^{m} \sum_{j=1}^{f} \alpha_{i j}(h) p_{i j}(h) \\
\text { subject to } & c_{i} \leq \mathbb{E}_{h} \sum_{j=1}^{f} \alpha_{i j}(h) q\left(h_{i j}, p_{i j}(h)\right), i=1, \ldots, m
\end{array}
$$

Here we have dropped the time indices $k$ from the variables $h_{k}$ since they are identically distributed over time. Finally we make a constraint qualification assumption that is typical in optimization theory, i.e., that a strictly feasible solution exists.

Assumption 3. There exist variables $\alpha^{\prime} \in \mathcal{A}$ and $p^{\prime} \in \mathcal{P}$ that satisfy the constraints of the optimization problem (20) with strict inequality, i.e.,

$$
c_{i}<\mathbb{E}_{h} \sum_{j=1}^{f} \alpha_{i j}^{\prime}(h) q\left(h_{i j}, p_{i j}^{\prime}(h)\right), \quad i=1, \ldots, m
$$

By the equivalence between problems (16) and (20), condition (21) can be interpreted as a feasibility/schedulability assumption for the shared wireless control system. It requires 
that there exist some channel-aware scheduling and power allocation such that the control performance requirements (13) of all control systems are met. This assumption however does not provide any information on how to find such a solution.

In the rest of the paper we examine problem (20), which is equivalent to the optimal scheduling and power allocation design for the shared wireless control architecture in Problem 1. Since this problem is feasible by Assumption 3 let us denote the optimal value by $P$ and an optimal solution pair by $\alpha^{*}(),. p^{*}($.$) . In the following section we characterize the$ form of the optimal solution and describe a methodology to obtain it.

Remark 2. Since $c_{i}$ is a required lower bound on the probability of successful transmission for system $i$, it must be that the value satisfies $c_{i}<1$. Equivalently the right hand side of (19) needs to be less than one for all values of $y$, which in turn is equivalent to $A_{c, i}^{T} P_{i} A_{c, i} \preceq \rho_{i} P_{i}$. This condition states that the closed-loop part of system (1) should satisfy the required decrease rate $\rho_{i}$ for the given quadratic Lyapunov function $V_{i}$, or in other words that if system $i$ transmits all the time the Lyapunov requirement is met.

\section{OPTIMAL SCHEDULING AND POWER ALLOCATION}

In this section we examine how the optimal scheduling and power allocation for the wireless control system can be recovered by considering the optimization problem in the dual domain. This allows us to develop an offline algorithm to solve the problem and provides an explicit characterization of the form of the optimal solution.

First let us derive the Lagrange dual problem of (20). Consider non-negative dual variables $\mu \in \mathbb{R}_{+}^{m}$ corresponding to each one of the $m$ constraints of (20). The Lagrangian then is defined as

$$
\begin{aligned}
& L(\alpha, p, \mu)=\mathbb{E}_{h} \sum_{i=1}^{m} \sum_{j=1}^{f} \alpha_{i j}(h) p_{i j}(h) \\
& +\sum_{i=1}^{m} \mu_{i}\left[c_{i}-\mathbb{E}_{h} \sum_{j=1}^{f} \alpha_{i j}(h) q\left(h_{i j}, p_{i j}(h)\right)\right],
\end{aligned}
$$

while the dual function is defined as

$$
g(\mu)=\inf _{\alpha, p \in(\mathcal{A}, \mathcal{P})} L(\alpha, p, \mu) .
$$

For future reference we also denote the set of functions $\alpha(),. p($.$) that minimize the Lagrangian at \mu$ by

$$
(\mathcal{A}, \mathcal{P})(\mu)=\underset{\alpha, p \in(\mathcal{A}, \mathcal{P})}{\operatorname{argmin}} L(\alpha, p, \mu),
$$

whenever the minimizers exist. This set might contain in general multiple solutions and we denote with $\alpha(\mu), p(\mu)$ an arbitrary element pair of the set. Since the pair itself is a function on $\mathcal{H}^{m}$ (cf. (10)), we denote the value of the pair at a point $h \in \mathcal{H}^{m}$ by $\alpha(\mu ; h), p(\mu ; h)$.

The Lagrange dual problem is defined as follows.

$$
D=\sup _{\mu \in \mathbb{R}_{+}^{m}} g(\mu) .
$$

According to Lagrange duality theory the optimal dual value $D$ is a lower bound on the optimal cost $P$ of problem (20). The following proposition however establishes a strong duality result $(D=P)$ for the problem under consideration and provides a relationship between the optimal primal and dual variables.

Proposition 1. Let Assumptions 1 and 3 hold. Let $P$ be the optimal value of the optimization problem (20) and $\left(\alpha^{*}, p^{*}\right)$ be an optimal solution, and let $D$ be the optimal value of the dual problem (25) and $\mu^{*}$ be an optimal solution. Then

(a) $P=D$ (strong duality)

(b) $\mu_{i}^{*}\left[c_{i}-\mathbb{E}_{h} \sum_{j=1}^{f} \alpha_{i j}^{*}(h) q\left(h_{i j}, p_{i j}^{*}(h)\right)\right]=0$ for all $i=$ $1, \ldots, m$ (complementary slackness)

(c) $\left(\alpha^{*}, p^{*}\right) \in(\mathcal{A}, \mathcal{P})\left(\mu^{*}\right)$

Proof: Statement (a) under assumptions 1 and 3 follows immediately from [21, Theorem 1] where a similar optimization setup is examined. The proof is omitted due to space limitations.

To show (b) observe that, by definition of the dual function in (23), at the point $\mu^{*}$ we have that

$$
g\left(\mu^{*}\right) \leq L\left(\alpha^{*}, p^{*}, \mu^{*}\right)
$$

Since $\mu^{*}$ is optimal for (25) and using part (a) we have for the left hand side of (26) that $g\left(\mu^{*}\right)=D=P$. On the other hand, the right hand side of (26), by the definition of the Lagrangian at (22), equals

$$
\begin{aligned}
& L\left(\alpha^{*}, p^{*}, \mu^{*}\right)=P \\
& +\sum_{i=1}^{m} \mu_{i}^{*}\left[c_{i}-\mathbb{E}_{h} \sum_{j=1}^{f} \alpha_{i j}^{*}(h) q\left(h_{i j}, p_{i j}^{*}(h)\right)\right],
\end{aligned}
$$

because the objective of (20) at the optimal solution $\left(\alpha^{*}, p^{*}\right)$ equals the optimal value $P$. These expressions for the left and right hand sides of the inequality in (26) therefore give

$$
P \leq P+\sum_{i=1}^{m} \mu_{i}^{*}\left[c_{i}-\mathbb{E}_{h} \sum_{j=1}^{f} \alpha_{i j}^{*}(h) q\left(h_{i j}, p_{i j}^{*}(h)\right)\right] .
$$

This implies that the sum on the right hand side is nonnegative. However all summands are non-positive, because $\mu^{*} \geq 0$ since it is feasible for the dual problem (25), and also the term in the brackets in (28) are non-positive because $\left(\alpha^{*}, p^{*}\right)$ are feasible for the primal problem (20). The only possibility then is that all summands in (28) are identically zero, which proves statement (b).

We have established that (28) holds with equality, so by tracing back our steps, we have that (26) holds with equality too, which, by the definition of the dual function on (23) translates to

$$
\inf _{\alpha, p \in(\mathcal{A}, \mathcal{P})} L\left(\alpha, p, \mu^{*}\right)=L\left(\alpha^{*}, p^{*}, \mu^{*}\right) .
$$

This verifies statement (c).

This proposition states that strong duality holds even though the original problem is not convex, regardless also of the form of the function $q(h, p)$ (Assumption 2 is not imposed). More importantly, part (c) suggests the possibility of recovering 
the optimal primal variables $\alpha^{*}, p^{*}$ by solving first for the optimal point $\mu^{*}$ in the dual problem. As we present next, this direction provides a significant advantage. The design of infinite-dimensional scheduling and power allocation policies that meet the control performance specifications in Problem 1 is reduced to the problem of determining finite-dimensional optimal dual variables. A technical caveat of Proposition 1(c) is that the optimal policies are included in a set which could in general contain other irrelevant policies. As we show next, Assumption 2 helps overcome this issue.

\section{A. Dual subgradient method}

To solve the dual problem in (25), that is, to maximize the dual function $g(\mu)$, we employ a dual projected subgradient algorithm [24, Ch. 8]. We first note that function $g(\mu)$ is concave, as a pointwise infimum over functions linear in $\mu$ (cf. (23)). A subgradient direction for $g(\mu)$ at any point $\mu \in \mathbb{R}_{+}^{m}$ is a vector, denoted here as $s(\mu) \in \mathbb{R}^{m}$, that satisfies

$$
g\left(\mu^{\prime}\right)-g(\mu) \leq\left(\mu^{\prime}-\mu\right)^{T} s(\mu) \text { for all } \mu^{\prime} \in \mathbb{R}_{+}^{m} .
$$

If we pick $\alpha(\mu), p(\mu) \in(\mathcal{A}, \mathcal{P})(\mu)$ by (24) then a subgradient $s(\mu)$ can be found as the constraint slack of the primal problem (20) evaluated at these points, i.e.,

$$
s_{i}(\mu)=c_{i}-\mathbb{E}_{h} \sum_{j=1}^{f} \alpha_{i j}(\mu ; h) q\left(h_{i j}, p_{i j}(\mu ; h)\right) .
$$

To show this observe that for any $\mu^{\prime}$ in general we have $g\left(\mu^{\prime}\right) \leq L\left(\alpha(\mu), p(\mu), \mu^{\prime}\right)$ by the definition of the dual function in (23). Subtracting $g(\mu)=L(\alpha(\mu), p(\mu), \mu)$ from both sides of this inequality and expanding the terms of the Lagrangian as in (22) we get

$$
\begin{aligned}
& g\left(\mu^{\prime}\right)-g(\mu) \leq \\
& \sum_{i=1}^{m}\left(\mu_{i}^{\prime}-\mu_{i}\right)\left[c_{i}-\mathbb{E}_{h} \sum_{j=1}^{f} \alpha_{i j}(\mu ; h) q\left(h_{i j}, p_{i j}(\mu ; h)\right)\right] .
\end{aligned}
$$

Comparing this with the property of the subgradient in (30), we verify that (31) indeed gives a subgradient direction. We also note for future reference that for any $\mu$ the subgradients are bounded because at the right hand side of (31) the term $c_{i}$ is bounded (cf.(19)) and the term in the expectation corresponds to a probability (cf.(11)).

A projected dual subgradient ascent method to maximize the concave dual function $g(\mu)$ consists of the following steps:

1) At iteration $t$ given $\mu(t)$ find primal optimizers of the Lagrangian at $\mu(t)$ according to (24),

$$
p(\mu(t)), \alpha(\mu(t)) \in(\mathcal{A}, \mathcal{P})(\mu(t))
$$

2) Evaluate the subgradient vector $s(\mu(t))$ by (31) and update the dual variables by an ascent step

$$
\mu(t+1)=[\mu(t)+\varepsilon(t) s(\mu(t))]_{+}
$$

where []$_{+}$denotes the projection on the non-negative orthant and $\varepsilon(t)>0$ is the stepsize.
The stepsizes are selected to be square summable but not summable, i.e.,

$$
\sum_{t \geq 1} \varepsilon(t)^{2}<\infty, \sum_{t \geq 1} \varepsilon(t)=\infty .
$$

Before stating the convergence properties of the algorithm, we note that in order to implement it we need an efficient way to compute primal Lagrange optimizers in (33) that solve (24). This problem also relates to our capability of finding the optimal primal variables of interest $\alpha^{*}, p^{*}$ as we have shown in Proposition 1(c). Hence we turn our focus to problem (24). A more convenient expression for the Lagrangian defined in (22) can be obtained by rearranging terms to get

$$
\begin{aligned}
& L(\alpha, p, \mu)=\mu^{T} c+ \\
& \mathbb{E}_{h} \sum_{i=1}^{m} \sum_{j=1}^{f} \alpha_{i j}(h)\left[p_{i j}(h)-\mu_{i} q\left(h_{i j}, p_{i j}(h)\right)\right] .
\end{aligned}
$$

This form provides a useful separation structure for the primal Lagrangian optimizers that we exploit in the following proposition.

Proposition 2. For any $\mu \in \mathbb{R}_{+}^{m}$ the following hold true:

(a) Solutions $\alpha(\mu), p(\mu) \in(\mathcal{A}, \mathcal{P})(\mu)$ of problem (24) can be obtained at each $h \in \mathcal{H}^{m}$ as

$$
\begin{aligned}
& p_{i j}(\mu ; h)=p_{i j}\left(\mu_{i} ; h_{i j}\right)=\underset{0 \leq p \leq p_{\max }}{\operatorname{argmin}} p-\mu_{i} q\left(h_{i j}, p\right) \\
& \text { for any } i=1, \ldots, m \text { and } j=1, \ldots, f \text {, and } \\
& \qquad \begin{array}{l}
\alpha(\mu ; h)=\underset{\alpha \in \mathbb{R}_{+}^{m \times f}}{\operatorname{argmin}} \sum_{i=1}^{m} \sum_{j=1}^{f} \alpha_{i j} \xi\left(h_{i j}, \mu_{i}\right) \\
\qquad \text { subject to } \sum_{i=1}^{m} \alpha_{i j} \leq 1, \sum_{j=1}^{f} \alpha_{i j} \leq 1
\end{array}
\end{aligned}
$$

where

$$
\xi\left(h_{i j}, \mu_{i}\right)=\min _{0 \leq p \leq p_{\max }} p-\mu_{i} q\left(h_{i j}, p\right) .
$$

(b) If Assumptions 1 and 2 hold, then for any solution $\alpha(\mu), p(\mu) \in(\mathcal{A}, \mathcal{P})(\mu)$ the vector $s(\mu)$ defined in (31) has a unique value.

Proof: See Appendix A

The first part of the proposition provides through equations (37) and (38) a method to obtain primal Lagrange optimizers that can be used in step (33) of the subgradient algorithm. Interestingly, the minimizing scheduling and power allocation decisions can be computed separately at each channel state value, hence significantly simplifying the computation. A further separability for the power allocation across systems and frequencies is revealed - see Remark 3.

The second part of the proposition relies on Assumption 2 to establish that the subgradient vector takes a unique value. Intuitively, Assumption 2 is used here to guarantee that the optimal Lagrange minimizers are almost surely unique. For example, Assumption 2(c) ensures this fact for the power minimizer in (37). This uniqueness allows a further characterization of the optimal primal variables than the set-characterization of 
Proposition 1(c). In particular, as we establish in the following theorem, the optimal scheduling and power allocation can be obtained by the Lagrangian minimization procedure presented in the first part of the above proposition.

Theorem 1 (Optimal Scheduling and Power Allocation). Consider the design of channel-aware scheduling and power allocation variables in Problem 1 for the shared wireless control architecture of Fig. 1, and let Assumptions 1, 2, 3 hold. Then optimal scheduling $\alpha^{*}$ and power allocation $p^{*}$ are obtained by (37)-(39) at a point $\mu^{*} \in \mathbb{R}_{+}^{m}$, which is an optimal solution of the dual problem (25). A point $\mu^{*}$ can be obtained by iterating (33)-(34), i.e., $\mu(t) \rightarrow \mu^{*}$, for stepsizes satisfying (35).

\section{Proof: See Appendix B}

The theorem characterizes the optimal scheduling and power allocation that meet the control performance specifications in our shared wireless control architecture - see the following remarks for more details about the form of the optimal policy. It is worth noting that the optimal policy need not be unique. More precisely, there might be many optimal dual solutions $\mu^{*}$, each corresponding to a different scheduling and power allocation policy according to the theorem. However all such policies will have the same objective value in (16).

The theorem also establishes a methodology to find the optimal communication policy by iterating (33)-(34). This can be viewed as an offline algorithm, and requires knowledge of the channel distribution. In the next section we develop an online algorithm that solves for the optimal communication policy based instead only on a random sequence of channel realizations observed during system execution.

Remark 3. According to Theorem 1, the optimal power allocation can be obtained at each channel value $h$ by solving (37) at the point $\mu^{*}$. In particular $p_{i j}^{*}(h)$ depends on the variables $\mu_{i}^{*}$ and $h_{i j}$ pertinent only to system $i$ and frequency $j$ and not on the whole vectors $\mu^{*}$ or $h$. This implies a decentralized power allocation among systems and frequencies, made explicit in (37) by the notation $p_{i j}\left(\mu_{i} ; h_{i j}\right)$. Similar separability results are also known in the context of resource allocation for wireless communication networks [21]. This fact can be intuitively understood from the shared wireless control architecture of Fig. 1, since each transmitter experiences different channel conditions and is responsible for an independent control task. Moreover, this optimal power allocation can be easily implemented in practice. The transmitter of each control system $i$ can store its value $\mu_{i}^{*}$ and adapt transmit power, whenever scheduled, based on the channel conditions it currently experiences. The optimal scheduling $\alpha^{*}(h)$ in (38), on the other hand, is centralized since it depends on the whole vector $\mu^{*}$ and all channel states $h$.

Remark 4. Determining the optimal scheduling in (38) is posed as a linear program by relaxing the integer constraints of $\Delta_{m, f}$ in (8). As mentioned in the proof of the proposition there is no loss in doing so, as the optimal solution to the linear program is integer. It is worth noting that (38) solves a standard assignment problem ${ }^{1}$. Besides the linear program presented here, integer programming algorithms with complexity polynomial in the number of systems $m$ and frequencies $f$ exist [25, Ch. 7]. In the special case of a single frequency $(f=1)$ the complexity of scheduling in (38) is linear in the number of systems $(O(m))$, since the scheduler looks for and schedules the system $i$ with the minimum value $\xi\left(h_{i}, \mu_{i}\right)$.

\section{ONLINE SCHEDULING AND POWER ALLOCATION}

The algorithm presented in the previous section to obtain optimal scheduling and power allocation for the shared wireless control system of Problem 1 is hard to implement in practice. In the primal step (33) one needs to obtain a solution pair $\alpha(h), p(h)$ for a continuum of channel variables $h \in \mathcal{H}^{m \times f}$, while for the dual step in (34) one needs to compute the subgradient direction $s(\mu)$ in (31) by integrating over the channel distribution $\phi$. A practical implementation would require drawing a large number of samples from $\phi$ and solving for primal variables at these samples to obtain an estimate of the actual subgradient direction. This is computationally intensive, does not scale for a large number of systems $m$ and frequencies $f$, while also in most cases of practical interest the channel distribution is not available.

These drawbacks motivate us to develop an online algorithm to solve Problem 1. The algorithm is a stochastic version of the primal/dual steps (33), (34) of the offline subgradient method and does not rely on availability of the channel distribution. In particular, suppose that at time $k$ a channel realization $h_{k}$ is observed, and the current power and scheduling decision are selected by solving (37)-(38) at the current $h_{k}$, i.e.,

$$
\begin{aligned}
p_{i j, k} & =p_{i j}\left(\mu_{i, k} ; h_{i j, k}\right), \quad i=1, \ldots, m, j=1, \ldots, f, \\
\alpha_{k} & =\alpha\left(\mu_{k} ; h_{k}\right) .
\end{aligned}
$$

Then in contrast to updating the dual variables $\mu_{k}$ by (34) after computing the vector (31), suppose only the current channel measurement and power/scheduling choices are used. In particular, suppose we compute

$$
s_{i, k}=c_{i}-\sum_{j=1}^{f} \alpha_{i j, k} q\left(h_{i j, k}, p_{i j, k}\right), \quad i=1, \ldots, m,
$$

and update the variables $\mu_{k}$ by

$$
\mu_{k+1}=\left[\mu_{k}+\varepsilon_{k} s_{k}\right]_{+}
$$

where []$_{+}$is the projection on the non-negative orthant and $\varepsilon_{k}>0$ is the stepsize.

To emphasize that this is an online algorithm we have explicitly indexed the variables with $k$ corresponding to real time slots. This procedure, summarized in Algorithm 1, gives scheduling and power variables $\left\{\alpha_{k}, p_{k}, k \geq 0\right\}$ as well as dual variables $\left\{\mu_{k}, k \geq 0\right\}$ which are random because they depend on the random observed channel sequence $\left\{h_{k}, k \geq 0\right\}$. The main difference compared to the subgradient algorithm of the previous section is that it follows random directions

\footnotetext{
${ }^{1}$ Technically the standard assignment problem requires equal number of systems and frequencies. This can be accomplished by introducing dummy systems or frequencies with zero values $\xi\left(h_{i j}, \mu_{i}\right)$.
} 


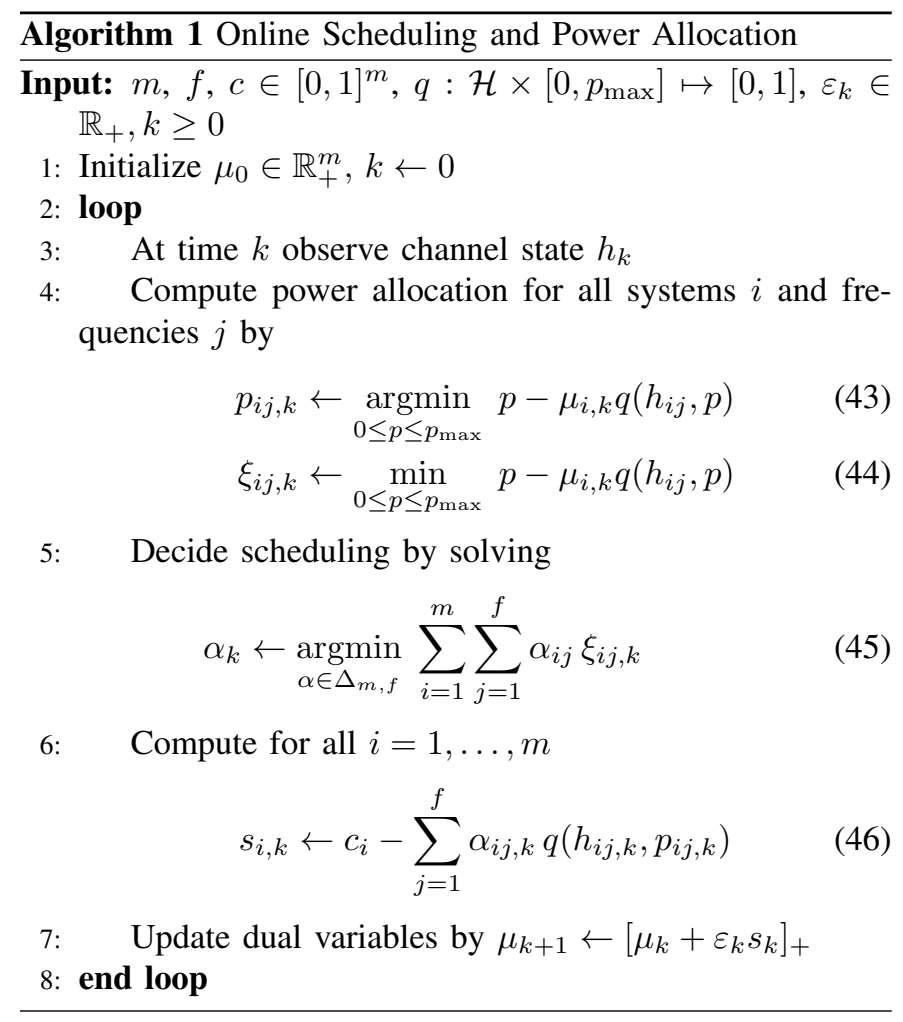

$s_{k}$ in (41) instead of the exact subgradient directions $s\left(\mu_{k}\right)$ by (31). Comparing these two expressions it is immediate that the expected value of $s_{k}$ coincides with the subgradient $s\left(\mu_{k}\right)$, so it is reasonable to conjecture that the online algorithm is expected to move towards the maximum of the dual function, as the subgradient method does. The following proposition indeed establishes convergence in a strong sense.

Proposition 3. Consider the optimization problem (20) and its dual derived in (25) and let Assumption 3 hold. Let a sequence $\mu_{k}, k \geq 0$ be obtained by steps (40)-(42) based on a sequence $\left\{h_{k}, k \geq 0\right\}$ of i.i.d. random variables with distribution $\phi$, and stepsizes $\varepsilon_{k}$ satisfying (35). Then almost surely we have that

$$
\lim _{k \rightarrow \infty} \mu_{k}=\mu^{*} \text {, and } \lim _{k \rightarrow \infty} g\left(\mu_{k}\right)=D
$$

where $\mu^{*}$ is an optimal solution of the dual problem and $D$ is the optimal value of the dual problem.

Proof: See Appendix C

The proposition states that the stochastic online algorithm yields a random sequence of dual variables $\mu_{k}$ that converges to the optimal point $\mu^{*}$ almost surely for any sequence of channel realizations that is observed. However the real problem of interest is the primal problem (20), or equivalently Problem 1. This is the problem of optimal design of scheduling and power allocation policies that satisfy the given Lyapunov performance requirements (13) for each control system $i$, while also minimizing the expected power expenditures of the communication process. Hence it is important to characterize how the control systems would actually perform if the communication variables are selected according to the proposed online algorithm. This characterization is provided in the following theorem.
Theorem 2 (Online Scheduling and Power Allocation). Consider a shared wireless control architecture composed of $m$ systems of the form (1), $f$ frequencies, and communication modeled by (9) depending on channel states $h_{k} \in \mathcal{H}^{m \times f}$ which are i.i.d. with distribution $\phi$, and scheduling and power allocation variables $\alpha_{k} \in \Delta_{m, f}, p_{k} \in\left[0, p_{\max }\right]^{m \times f}$. Also consider given quadratic Lyapunov performance requirements (13) for each system and let Assumptions 1, 2, 3 hold. If $\alpha_{k}, p_{k}$ are chosen according to (40)-(42), then almost surely with respect to the channel sequence $\left\{h_{k}, k \geq 0\right\}$ the control performances for all systems $i=1, \ldots, m$ satisfy

$$
\begin{aligned}
\limsup _{k \rightarrow \infty} \mathbb{E}\left[V_{i}\left(x_{i, k+1}\right) \mid x_{i, k}\right. & \left.=x_{i}, h_{0}, \ldots, h_{k-1}\right] \\
& \leq \rho_{i} V_{i}\left(x_{i}\right)+\operatorname{Tr}\left(P_{i} W_{i}\right),
\end{aligned}
$$

for any state values $x_{i} \in \mathbb{R}^{n_{i}}$. In addition, the power consumption almost surely satisfies

$$
\limsup _{k \rightarrow \infty} \mathbb{E}\left[\sum_{i=1}^{m} \sum_{j=1}^{f} \alpha_{i j, k} p_{i j, k} \mid h_{0}, \ldots, h_{k-1}\right] \leq P
$$

where $P$ is the optimal value of the optimization problem (16).

Proof: See Appendix D.

According to the theorem the scheduling and power allocation variables selected by the online algorithm lead in the limit to the desired Lyapunov requirements for all control systems and to the optimal power expenditure, for almost all channel sequences. We can also establish the following corollary.

Corollary 1. Consider the setup of Theorem 2. Then for any positive constant $\delta>0$ there exists a time step $N$ such that for all times $k \geq N$ we have that

$$
\mathbb{E}\left[V_{i}\left(x_{i, k+1}\right) \mid x_{i, k}\right] \leq\left(\rho_{i}+\delta\right) V_{i}\left(x_{i, k}\right)+\operatorname{Tr}\left(P_{i} W_{i}\right)
$$

for any possible value of plant states $x_{i, k} \in \mathbb{R}^{n_{i}}$ and for all systems $i=1, \ldots, m$.

Proof: See Appendix E.

Recall that we initially asked for a communication design that guarantees expected control performance requirements at each time step $k$ in (13). According to the above corollary our online policy approximately satisfy this. After a sufficiently long time horizon the expected decrease rates of all Lyapunov functions get arbitrarily close to the desired ones. Before proceeding to simulations of the stochastic online algorithm, we present an intuitive interpretation of the algorithm from an economic resource allocation point of view.

\section{A. Pricing interpretation of online scheduling and power allocation algorithm}

In this section we provide an interpretation of the problem variables as well at the online Algorithm 1 in economic terms. In particular we may view each transmitter in the wireless control architecture as an agent that utilizes some scarce resource, namely transmit power, to produce some 'good', namely the probability of successfully transmitting and closing the corresponding control loop. Our development in Section II-A shows that each closed loop has a Lyapunov control 
performance requirement (cf. (13)) that can be translated as requiring $c_{i}$ units of good (cf. (19)). Under this view, the dual variables $\mu_{i}$ can be interpreted as the 'unit price' at which each agent can 'sell' the produced good. In this context the role of Algorithm 1 is to determine unit prices such that all demand levels $c_{i}$ are met and in the most profitable manner from the agents' perspective.

More specifically, consider a time step $k$ where prices are set to $\mu_{k}$ and the current channel conditions are described by $h_{k}$. If agent $i$ gets access to the channel at frequency $j$, the agent can spend an amount $p_{i j, k}$ to produce $q\left(h_{i j, k}, p_{i j, k}\right)$ units of good, which can be sold at a price of $\mu_{i, k}$ per unit. In this case the total profit for the agent can be expressed as

$$
\mu_{i, k} q\left(h_{i j, k}, p_{i j, k}\right)-p_{i j, k}
$$

i.e., the difference between the total revenue $\mu_{i, k} q\left(h_{i j, k}, p_{i j, k}\right)$ and the total cost $p_{i j, k}$. The optimal resource allocation $p_{i j, k}$ is the one maximizing the profit (51), matching exactly the optimization over power provided in (43). The optimal profit if agent $i$ gets access to the channel at frequency $j$ under conditions $h_{i j, k}$ equals $-\xi_{i j, k}$ given in (44).

Then the role of the scheduler is to opportunistically assign agents to the available frequencies in a way that maximizes the total aggregated profit. In particular the scheduler observes current conditions $h_{i j, k}$ for all agents $i$ and frequencies $j$, computes the possible profit $-\xi i j, k$ of all agent/frequency pairs, and searches for the scheduling $\alpha \in \Delta_{m, f}$ defined by (8) that maximizes the total profit

$$
\sum_{i, j} \alpha_{i j}(-\xi i j, k)
$$

aggregated over all agents. This optimal scheduling matches the one implemented by Algorithm 1 (cf. line (45)).

After the current scheduling $\alpha_{k}$ and power $p_{k}$ decisions have been made, the unit prices $\mu_{k+1}$ for the next step are adjusted depending on the current production levels. If the production for system $i$ exceeds the required level $c_{i}$, i.e., $s_{i, k}<0$ in (41), then the unit price for system $i$ is reduced to $\mu_{i, k}+\varepsilon_{k} s_{i, k}$ (cf. line 7 in Algorithm 1). If on the other hand the production for system $i$ does not meet $c_{i}$, i.e., $s_{i, k}>0$, then the unit price $i$ increases to $\mu_{i, k}+\varepsilon_{k} s_{i, k}$.

According to Proposition 3 and Theorem 2 the online algorithm converges almost surely to the optimal prices $\mu^{*}$, under which the expected production meets demand, where expectation is with respect to the channel conditions. Moreover the expected total production cost (the objective of problem (20)) becomes optimal in the limit.

Note however that Theorem 2 does not provide theoretical guarantees on how fast the solution converges to the optimal one. We discuss this issue along with other limitations of the algorithm in Section VI. In the following section we present simulations verifying our theoretical results, and also indicating that the convergence of the algorithm is relatively fast so that online control performance is not severely affected.

\section{Numerical Simulations}

\section{A. Opportunistic nature of scheduling and power allocation}

We first illustrate through simulations the opportunistic nature of the resource allocation mechanism for wireless control systems obtained in Section III, in particular how scheduling and power decisions adapt appropriately to channel conditions to meet the control performance goals. Moreover we compare the resulting performance with other simple non channeladaptive allocation mechanisms. Recall that by solving the auxiliary problems (19), control systems with vector states are converted to scalar constraints in optimization problem (20). Hence without loss of generality we present an example with scalar control systems.

Consider a heating system application controlling the temperature in two independent rooms of a building. Assuming the wireless control architecture of Fig. 1 with $m=2$, wireless sensors transmit the temperatures of each room to a central location (the access point in Fig. 1) responsible for adjusting the heating in the rooms. For simplicity suppose both systems have identical dynamics of the form (1) with state $x_{i, k}$ denoting the difference between current and some desired temperature for room $i$. In particular suppose that when system $i$ transmits $\left(\gamma_{i, k}=1\right)$, heating is activated for system $i$ and results in stable dynamics $A_{c, i}=0.4$ in (1). Otherwise if $\gamma_{i, k}=0$ the system is open loop unstable with $A_{o, i}=1.1$ in (1), e.g., because heating is deactivated.

For simplicity we assume there is one $(f=1)$ available frequency and for symmetry let channel states $h_{1, k}$ and $h_{2, k}$ be independent for each system, both having an exponential distribution with mean 1 . The function $q(h, p)$ is shown in Fig. 2. For these scalar systems it suffices to consider Lyapunov functions $V_{i}(x)=x^{2}$. We require then that system 1 guarantees a high Lyapunov decrease $\rho_{1}=0.75$ rate according to (13), while system 2 only requires $\rho_{2}=0.90$. For these choices we get a higher required success of transmission $c_{1} \approx 0.44$ according to (19) for system 1 , compared to a lower $c_{2} \approx 0.30$ of system 2 .

After solving problem (20) offline according to the subgradient method of Section III, the optimal channel-aware scheduling and power allocation variables are depicted in Fig. 3 and Fig. 4 respectively. We observe in Fig. 3 that System 1, which requires higher transmission success $c_{1}$, is scheduled to transmit for most values of the channel states $h_{1}, h_{2}$. System 2, which has a lower requirement, is scheduled only if its channel $h_{2}$ is sufficiently favorable and system 1 experiences an adverse channel $h_{1}$. This illustrates how the scheduler exploits opportunistically the channel conditions to select which system will transmit to close the loop, in order to meet the Lyapunov constraints in a power efficient manner. We also note that when both systems experience very adverse channels the scheduling decision becomes irrelevant because, as we will see in Fig. 4, the optimal transmit powers then are zero (no transmission).

The optimal power allocation is decentralized as we noted in Remark 3 , i.e., the transmit power $p_{i}$ for system $i$ depends only on the channel $h_{i}$ that system $i$ experiences, and thus we plot in Fig. 4 the power allocation for both systems on same axes. 


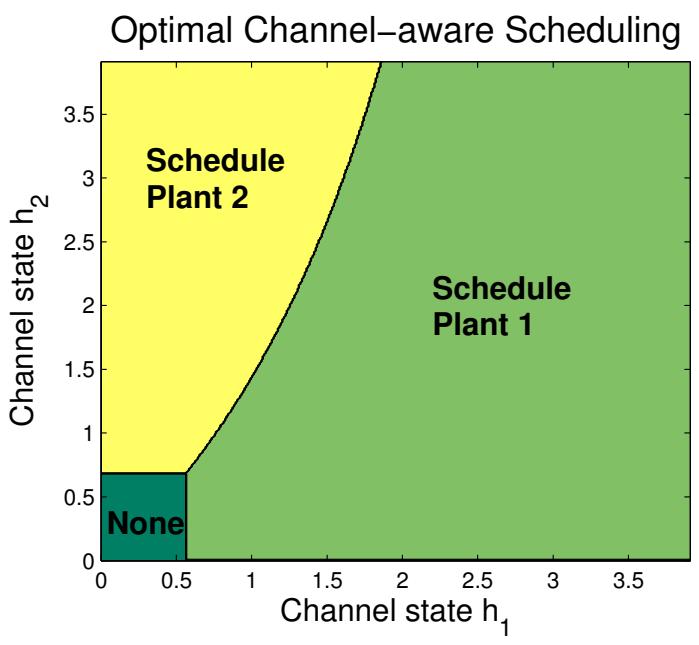

Fig. 3. Optimal channel-aware scheduling for the example presented in Section V. System 1 has a harder Lyapunov decrease rate requirement and is scheduled to transmit for most observed channel states $h_{1}, h_{2}$. System 2 is scheduled only if its channel conditions $h_{2}$ are much more favorable that those of system 1 . When both channels are very adverse systems select zero transmit powers so scheduling is irrelevant.

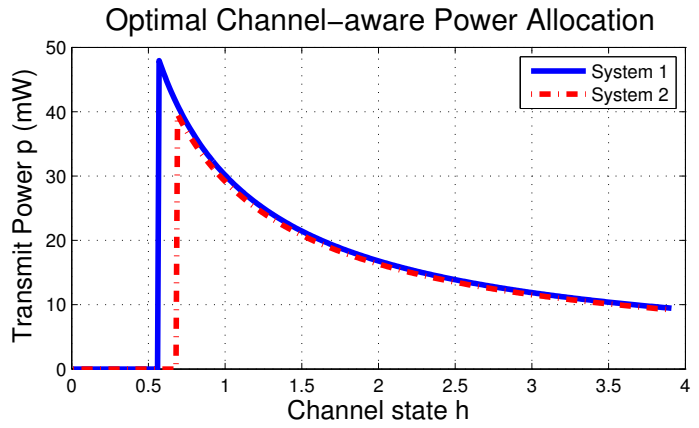

Fig. 4. Optimal channel-aware power allocation for the example presented in Section V. Under adverse channel conditions systems do not transmit. The channel threshold for transmission for system 1 is lower than that of system 2 because the former has a higher Lyapunov decrease rate requirement. System 1 also requires higher transmit power.

For both systems, when the channel conditions are adverse it is not worth to spend transmit power. System 1, which has a more demanding control constraint, requires in general higher transmit power since, as we saw in Fig. 3, it is scheduled to transmit even under adverse channel conditions. This is also captured in the expected power consumption of each system computed numerically as $\mathbb{E}_{h} \alpha_{1}^{*}(h) p_{1}^{*}\left(h_{1}\right) \approx 11 \mathrm{~mW}$ and $\mathbb{E}_{h} \alpha_{2}^{*}(h) p_{2}^{*}\left(h_{2}\right) \approx 6.5 \mathrm{~mW}$. The minimum total power budget required to meet the control objectives then is approximately $17.5 \mathrm{~mW}$.

To demonstrate the power savings obtained by the opportunistic resource allocation we compare to a simple nonchannel-aware communication mechanism. In particular suppose that at each step a system is chosen randomly to access the channel/frequency. With a slight abuse of notation suppose systems 1 and 2 are chosen with probabilities $\alpha_{1}$ and $\alpha_{2}=1-\alpha_{1}$ respectively. When a system is selected, we suppose it transmits with a constant power level $p_{c}$. The control performance requirements (cf. (20)) in this case

\begin{tabular}{l|l|l|l|l|l} 
& $\begin{array}{l}\text { Control } \\
\text { objective } \\
\rho\end{array}$ & $\begin{array}{l}\text { Mean } \\
\text { Fading } \\
h_{i 1}\end{array}$ & $\begin{array}{l}\text { Mean } \\
\text { Fading } \\
h_{i 2}\end{array}$ & $\begin{array}{l}\text { Transmit } \\
\text { Rate at } \\
\text { Freq. 1 }\end{array}$ & $\begin{array}{l}\text { Transmit } \\
\text { Rate at } \\
\text { Freq. 2 }\end{array}$ \\
\hline Plant 1 & 0.75 & 1 & 1 & 0.25 & 0.23 \\
\hline Plant 2 & 0.9 & 1 & 1 & 0.18 & 0.15 \\
\hline Plant 3 & 0.9 & 1 & 2 & 0.07 & 0.25
\end{tabular}

SYSTEM PARAMETERS \& ONLINE TRANSMISSION RATES

become $\alpha_{i} \mathbb{E}_{h_{i}} q\left(h_{i} p_{c}\right) \geq c_{i}$ for $i=1,2$ and the total power cost is $\left(\alpha_{1}+\alpha_{2}\right) p_{c}=p_{c}$. We briefly comment then on possible designs for $\alpha_{1}$ and $p_{c}$.

First, observe from the channel-aware design in Fig. 4 that a system never transmits with power level larger than $50 \mathrm{~mW}$. Suppose then we select the power budget $p_{c}=50 \mathrm{~mW}$. It turns out that the two control performance requirements cannot be achieved in this case, because we compute

$$
\sum_{i=1,2} \alpha_{i} \mathbb{E}_{h_{i}} q\left(h_{i} p_{c}\right)=\mathbb{E}_{h_{i}} q\left(h_{i} p_{c}\right) \approx 0.65<c_{1}+c_{2} \approx 0.74
$$

meaning that the constraints are infeasible. Searching numerically for a value $p_{c}$ where the random access scheme meets the control objectives, we find $p_{c} \approx 73 \mathrm{~mW}$. Contrasting this amount with the optimal power budget of the opportunistic case above, in this example the channel-aware resource allocation succeeded almost a $80 \%$ decrease in power budget compared to a not channel-aware random access scheme.

\section{B. Stochastic online scheduling and power allocation}

Next we implement the stochastic online algorithm of Section IV in a setup with three $(m=3)$ control loops sharing two $(f=2)$ frequencies. For example consider again the room heating system of the previous section including three rooms/systems with identical dynamics, $A_{o, i}=1.1$ and $A_{c, i}=0.4$ as before. We set the desired Lyapunov decrease rates as $\rho_{1}=0.75, \rho_{2}=\rho_{3}=0.9$, implying that system 1 is more demanding in communication resources. We assume channel states $h_{i j}$ are independent across systems $i$ and frequencies $j$, and have exponential distributions with means given in Table I. In particular we model that system 2 experiences better channel quality (higher channel fading gain) in frequency 2 .

The evolution of the dual variables $\mu_{k}$ during Algorith 1 is shown in Fig. 5. After a number of iterations (time $k$ in this example corresponds to seconds) they remain in a small neighborhood around the optimal $\mu^{*}$, as anticipated by the theoretical a.s. convergence in Prop. 3. Consequently, the scheduling and power allocation decisions taken online are almost feasible for the constraints of problem (20) after a number of iterations. We observe that the dual variable corresponding to system 1 is the largest, consistent with the fact that it has a harder control requirement to meet. Using the economic interpretation of Section IV-A about the dual variables, the price at which agent 1 can sell its produced good is higher, giving the incentive to schedule agent 1 to produce more often. On the other hand, systems 2 and 3 have the same control requirements but the dual variable for system 2 is larger. The reason is that system 2 experiences worse 
Online Evolution of Dual Variables

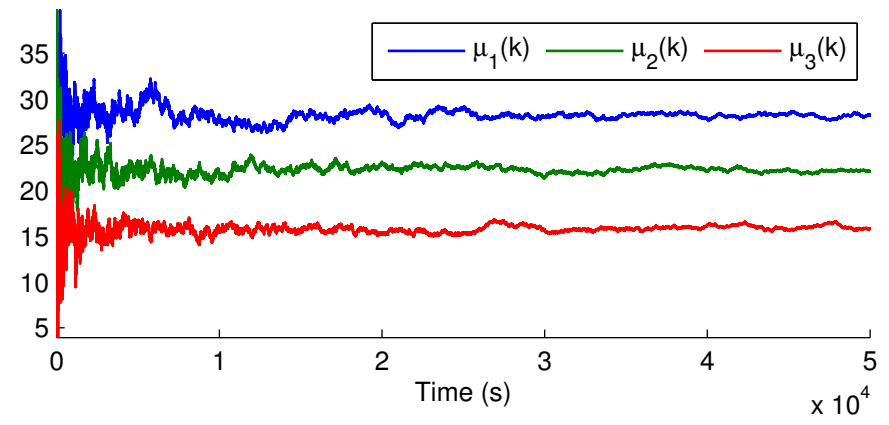

Fig. 5. After a number of steps during the online algorithm the dual variables $\mu_{k}$ remain in a neighborhood around the optimal $\mu^{*}$.

channel conditions than system 3 (cf. Table I), which imply higher required transmit power, or in economic terms a higher production cost in (51). By setting a higher selling price $\mu_{2}$, system 2 becomes profitable enough so that it is scheduled to produce at a sufficient rate to meet the requirement.

In Table I we show the average transmission rates that the online algorithm selected during system execution. In particular we evaluate the average number of time slots where each system $i$ was selected to transmit (with a positive power level) at each frequency $j$ as $1 / N \sum_{k=1}^{N} \alpha_{i j, k} \mathbb{I}\left(p_{i j, k}>0\right)$. System 3 was scheduled mainly at frequency 2, exploiting its better channel quality. This forced systems 1 and 2 to use frequency 1 more often. Also system 1, which has higher control requirement, transmitted more often than the other systems. We note that this behavior resulted from the online algorithm using only an observed channel sequence, not any prior knowledge on the channel quality distribution.

Finally, we examine the evolution of the three heating control systems when the online algorithm is employed for scheduling and power decisions. Suppose that for all systems $i$ the states $x_{i}$, which measure deviations from reference room temperatures, are perturbed by disturbances $w_{i, k}$ as in (1), which we model as independent Gaussian with mean zero and variance $W_{i}=1$ (at some normalized units of temperature). We plot in Fig. 6 the evolution of the empirical quadratic averages $1 / N \sum_{k=1}^{N} x_{i, k}^{2}$. Recall that when the Lyapunov condition (13) is satisfied, we get from (14) that the expected limit quadratic costs are bounded by $W_{i} /\left(1-\rho_{i}\right)$. We observe from Fig. 6 that after some initial transient the online communication algorithm keeps the empirical average quadratic costs close to the theoretical required upper bounds.

\section{DISCUSSION AND CONCLUSIONS}

This paper considers opportunistic channel-aware schedulers for wireless control systems with multiple loops closing over a shared wireless medium. We develop a suitable stochastic optimization formulation, and design scheduling and transmit power policies that minimize the total expected power expenditures while guaranteeing that given Lyapunov functions for each control system exhibit desired expexted decrease rates for stability and performance. We develop an offline optimization algorithm to solve the problem, as well

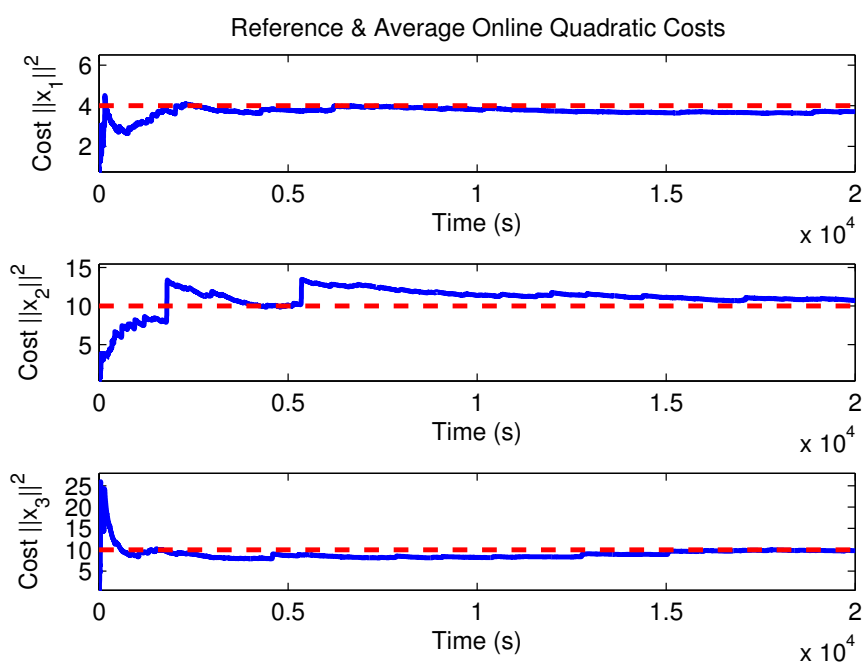

Fig. 6. Average quadratic costs during the online scheduling and power allocation algorithm. The stochastic algorithm keeps the average quadratic cost of each control system close to the upper bound of the limit expected cost, shown with dashed lines, induced theoretically by the required Lyapunov decrease rates.

as an online communication algorithm that converges to the optimal based on random observed channel sequences. An extension of the present paper for scheduling inter-dependent control tasks is considered in [26].

The proposed online algorithm guarantees almost sure convergence, but a theoretical characterization of the convergence rate is not provided. The online algorithm also uses decreasing stepsizes, which limits the adaptability to an environment with non-stationary channel distributions. These issues will be the focus of future work. Moreover, our methodology relies on the existence of feasible scheduling and power allocation policies (Assumption 3), hence sufficient system conditions for this to hold would be desirable. A different research direction is to include, apart from channel states, the measured plant system states when making the scheduling decisions, as in, e.g., [11]-[13], or in the single-loop power management paradigm of [22]. Furthermore, the problem of joint scheduling and controller design, which would allow control adapting to communication events as in [3], poses new research challenges. Finally, the present development can serve as a framework for examining decentralized channel access mechanisms, such as random access [18, Ch. 14], which would obviate the use of a centralized scheduler in Fig. 1.

\section{APPENDIX}

\section{A. Proof of Proposition $2^{2}$}

We first show part (a) of the proposition. Consider the problem of minimizing the Lagrangian as given at the form (36) over variables $\alpha(),. p($.$) for some \mu \in \mathbb{R}_{+}^{m}$. Since $\mu^{T} c$ is constant the problem is equivalent to

$$
\inf _{\alpha, p \in(\mathcal{A}, \mathcal{P})} \mathbb{E}_{h} \sum_{i, j} \alpha_{i j}(h)\left[p_{i j}(h)-\mu_{i} q\left(h_{i j}, p_{i j}(h)\right)\right] .
$$

\footnotetext{
${ }^{2}$ Within this proof we denote $\sum_{i=1}^{m} \sum_{j=1}^{f}$ as $\sum_{i, j}$ for compactness.
} 
Without loss of generality we can exchange the expectation over $h$ and the minimization over functions $\alpha(),. p($.$) in (54)$ to equivalently solve for each $h \in \mathcal{H}^{m \times f}$

$$
\inf _{\substack{\alpha(h) \in \Delta_{m, f} \\ p(h) \in\left[0, p_{\max }\right]^{m \times f}}} \sum_{i, j} \alpha_{i j}(h)\left[p_{i j}(h)-\mu_{i} q\left(h_{i j}, p_{i j}(h)\right)\right]
$$

This step is valid because any pair of functions $\alpha, p$ that does not minimize the objective in (55) on a set of values of variables $h$ with $\phi$-positive measure must yield a strictly larger expected value in the objective of (54). In other words, the minimizers of (54) can only differ from the minimizers of (55) at a set of values for $h$ with measure zero.

Then note that at any $h \in \mathcal{H}^{m \times f}$ and any choice for the variable $\alpha(h)$ we have that $\alpha_{i j}(h) \geq 0$. Hence the optimization over $p(h)$ in (55) can be rearranged to

$$
\begin{aligned}
\inf _{\alpha(h) \in \Delta_{m, f}} & \sum_{i, j} \alpha_{i j}(h) \\
& \inf _{p_{i j}(h) \in\left[0, p_{\max }\right]} p_{i j}(h)-\mu_{i} q\left(h_{i j}, p_{i j}(h)\right) .
\end{aligned}
$$

The optimization over power variables $p_{i, j}(h)$ in this expression corresponds exactly to (37). Using the notation introduced in (39), the minimization over scheduling variables $\alpha(h)$ in (56) becomes

$$
\inf _{\alpha(h) \in \Delta_{m, f}} \sum_{i, j} \alpha_{i j}(h) \xi\left(h_{i j}, \mu_{i}\right),
$$

The expression given in (38) is obtained by relaxing the integer constraint $\alpha_{i j} \in\{0,1\}$ of the set $\Delta_{m, f}$ (cf.(8)) in problem (57) with $\alpha_{i j} \geq 0$. The resulting problem (38) is a linear program, but the optimal solution will be integer (see, e.g., [25, Th. 7.5]) and feasible with respect to $\Delta_{m, f}$.

Now let us prove part (b) of the proposition. We need to show that any pair $\alpha(\mu), p(\mu)$, which are functions of $h$, that solves (54) gives a unique evaluation of $s(\mu)$ given in (31). Since $s_{i}(\mu)$ involves integrating the term

$$
\sum_{j=1}^{f} \alpha_{i j}(\mu ; h) q\left(h_{i j}, p_{i j}(\mu ; h)\right)
$$

with respect to the distribution $\phi$ of $h \in \mathcal{H}^{m \times f}$, it suffices to show that (58) is unique $\phi$-a.s.

By the argument presented already, minimizing (54) is a.s. equivalent to minimizing (55). The latter is again equivalent to the problem (56) since all $\alpha_{i j}(h) \geq 0$. Note that the only case where the optimizers in (55) can differ from the ones obtained in (56) is if $\alpha_{i j}(\mu ; h)=0$ for some $i, j$ is optimal at some values $h \in \mathcal{H}^{m \times f}$ and the power minimizer $p_{i j}(\mu ; h)$ in (55) can be chosen arbitrarily. But this does not affect the computation of $s_{i}(\mu)$ since (58) will equal zero. Hence we only need to show that the minimizers $\alpha(\mu ; h), p(\mu ; h)$ in (56) imply a.s. uniqueness of (58).

For values of $h$ where the minimizers $\alpha(\mu ; h), p(\mu ; h)$ of problem (56) are unique it is immediate that (58) has a unique value, hence we only need to consider $h$ where the minimizers are not unique. By Assumption 2(c) the minimizer $p(\mu ; h)$, which is given in (37), is unique for almost all $h$, therefore we only need to focus on the set of values for $h$ where the minimizer $\alpha(\mu ; h)$, described by (38), is not unique.

Let us denote by $E$ the set of interest, i.e., the set of $h \in$ $\mathcal{H}^{m \times f}$ where $\alpha(\mu ; h)$ in (38) is not unique. By considering all possible pairs of multiple solutions $\alpha^{\prime} \neq \alpha^{\prime \prime}$ in the finite set $\Delta_{m, f}$, we can rewrite $E$ as a union $E=\bigcup_{\alpha^{\prime} \neq \alpha^{\prime \prime} \in \Delta_{m, f}} E_{\alpha^{\prime}, \alpha^{\prime \prime}}$ where $E_{\alpha^{\prime}, \alpha^{\prime \prime}} \subseteq \mathcal{H}^{m \times f}$ such that

$$
h \in E_{\alpha^{\prime}, \alpha^{\prime \prime}} \Leftrightarrow \alpha^{\prime}, \alpha^{\prime \prime} \in \underset{\alpha \in \Delta_{m, f}}{\operatorname{argmin}} \sum_{i, j} \alpha_{i j} \xi\left(h_{i j}, \mu_{i}\right) .
$$

In other words, the set $E_{\alpha^{\prime}, \alpha^{\prime \prime}}$ is the set of values $h$ where both $\alpha^{\prime}, \alpha^{\prime \prime}$ are optimal for (38). The rest of the proof shows that on any $E_{\alpha^{\prime}, \alpha^{\prime \prime}}$ the value of (58) is almost surely unique.

The set $E_{\alpha^{\prime}, \alpha^{\prime \prime}}$ depends on the shape of the function $\xi$ defined in (39), so next we point out two properties of $\xi\left(h_{i j}, \mu_{i}\right)$.

Fact 1: For almost all $h_{i j}$ where the optimal value of problem (39) is $\xi\left(h_{i j}, \mu_{i}\right)=0$, the optimal solution is unique and equals $p_{i j}(\mu ; h)=0$.

Proof of Fact 1: First we note that for any $h_{i j}$, the choice $p=0$ is feasible for problem (39) and by Assumption 2(a) it gives an objective $p-\mu_{i} q\left(h_{i j}, p\right)=0$. So whenever the optimal value of problem (39) is 0 , then $p=0$ is an optimal solution. This optimal solution is unique for almost all $h_{i j}$ because of Assumption 2(c).

Fact 2: If at some $h_{i j}$ the optimal value of problem (39) is $\xi\left(h_{i j}, \mu_{i}\right)<0$, then for $h_{i j}^{\prime}>h_{i j}$ we have that $\xi\left(h_{i j}^{\prime}, \mu_{i}\right)<$ $\xi\left(h_{i j}, \mu_{i}\right)$.

Proof of Fact 2: First note that at the given $h_{i j}$ it must be that the optimal solution $p_{i j}(\mu ; h)$ of problem (39) satisfies $q\left(h_{i j}, p_{i j}(\mu ; h)\right)>0$. This is true because otherwise $q\left(h_{i j}, p_{i j}(\mu ; h)\right)=0$ implies $\xi\left(h_{i j}, \mu_{i}\right)=p_{i j}(\mu ; h) \geq 0$. Second by Assumption 2(b) when $q()>$.0 , it is strictly increasing in its argument. Thus we have

$$
\begin{aligned}
& \xi\left(h_{i j}, \mu_{i}\right)=p_{i j}(\mu ; h)-\mu_{i} q\left(h_{i j}, p_{i j}(\mu ; h)\right) \\
& >p_{i j}(\mu ; h)-\mu_{i} q\left(h_{i j}^{\prime}, p_{i j}(\mu ; h)\right) \geq \xi\left(h_{i j}^{\prime}, \mu_{i}\right)
\end{aligned}
$$

for $h_{i j}^{\prime}>h_{i j}$.

Let us now fix some $\alpha^{\prime} \neq \alpha^{\prime \prime} \in \Delta_{m, f}$ and consider the set $E_{\alpha^{\prime}, \alpha^{\prime \prime}}$. Pick indices $\imath, \jmath$ where $\alpha^{\prime}, \alpha^{\prime \prime}$ differ, i.e., without loss of generality, $\alpha_{\imath, \jmath}^{\prime}=1, \alpha_{\imath, \jmath}^{\prime \prime}=0$. Consider first the case of $h \in E_{\alpha^{\prime}, \alpha^{\prime \prime}}$ where $\xi\left(h_{\imath, \jmath}, \mu_{\imath}\right)=0$. By Fact 1 above we know that this implies $p_{\imath, j}(\mu ; h)=0$ is almost surely the unique optimizer of (37). But in that case $q\left(h_{\imath, \jmath}, p_{\imath, \jmath}(\mu ; h)\right)=0$, and the choice of $\alpha_{\imath, \jmath}(h)$ does not affect the value of (58), which is zero.

Second, we examine the set $h \in E_{\alpha^{\prime}, \alpha^{\prime \prime}}$ where $\xi\left(h_{\imath \jmath}, \mu_{\imath}\right)<$ 0 . We will show that this event happens with $\phi$-probability zero. In particular by Assumption $1 \phi$ has a probability density function on $\mathcal{H}^{m \times f}$, or more formally $\phi$ is absolutely continuous with respect to the Lebesgue measure on $\mathcal{H}^{m \times f}$. Hence to show that the discussed event has $\phi$-measure zero, it suffices to show that it has Lebesgue measure zero. Note that 
we can upper bound the set as follows

$$
\begin{aligned}
& E_{\alpha^{\prime}, \alpha^{\prime \prime}} \bigcap\left\{h: \xi\left(h_{\imath \jmath}, \mu_{\imath}\right)<0\right\} \\
& \subseteq\left\{h: \sum_{i, j}\left(\alpha_{i j}^{\prime \prime}-\alpha_{i j}^{\prime}\right) \xi\left(h_{i j}, \mu_{i}\right)=0, \quad \xi\left(h_{\imath \jmath}, \mu_{\imath}\right)<0\right\} \\
& =\left\{h: \sum_{i \neq \imath, j \neq \jmath}\left(\alpha_{i j}^{\prime \prime}-\alpha_{i j}^{\prime}\right) \xi\left(h_{i j}, \mu_{i}\right)=\xi\left(h_{\imath \jmath}, \mu_{\imath}\right)<0\right\}
\end{aligned}
$$

The subset in the first step is justified from the fact that, in contrary to the definition of $E_{\alpha^{\prime}, \alpha^{\prime \prime}}$ in (59), we do not take $\alpha^{\prime}, \alpha^{\prime \prime}$ to be optimal for problem (38). We only require that they yield the same objective in the problem. The second step follows by the appropriately selected indices $\imath, \jmath$.

We will now argue that the last set in (61) has Lebesgue measure zero. If we fix the values of all the variables/coordinates $h_{i j}, i \neq \imath, j \neq \jmath$, there is at most one value for the variable/coordinate $h_{\imath \jmath}$ that belongs in the set. The reason is that for values of the $h_{\imath \jmath}$ coordinate where $\xi\left(h_{\imath \jmath}, \mu_{\imath}\right)<0$, Fact 2 above states that $\xi\left(h_{\imath \jmath}, \mu_{\imath}\right)$ is strictly monotonic in $h_{\imath \jmath}$. Hence there can be at most one value $h_{\imath \jmath}$ that equals the sum within the last set of (61). This means that the last set in (61) can be equivalently described by a mapping from an $m \cdot f-1$ dimensional space to the space $\mathcal{H}^{m \times f}$, or in other words it is a lower-dimensional subset of $\mathcal{H}^{m \times f}$. Hence it has Lebesgue measure zero. This implies that the first set in (61) has Lebesgue (and $\phi$ ) measure zero as well.

The above procedure can be iterated for any pair $\alpha^{\prime}, \alpha^{\prime \prime}$ to conclude that in their union set $E$ the value of the subgradient vector is almost surely unique.

\section{B. Proof of Theorem 1}

Let $\mu^{*}$ be an optimal solution of the dual problem (25). First, we argue that every pair $\alpha\left(\mu^{*}\right), p\left(\mu^{*}\right)$ chosen from the set of Lagrangian minimizers $(\mathcal{A}, \mathcal{P})\left(\mu^{*}\right)$ at the point $\mu^{*}$ (cf. (24)) is an optimal solution to primal Problem 1 (equivalently (20)). Under Assumptions 1 and 2, Proposition 2(b) states that the vector $s\left(\mu^{*}\right)$ in (31) has the same value at any chosen pair $\alpha\left(\mu^{*}\right), p\left(\mu^{*}\right)$. Since $s\left(\mu^{*}\right)$ is also the constraint slack of the chosen pair in the primal problem (20), then any Lagrange optimizers $\alpha\left(\mu^{*}\right), p\left(\mu^{*}\right)$ have the same constraint slack. Moreover, under Assumptions 1 and 3, Proposition 1(c) states that the optimal primal variables $\alpha^{*}, p^{*}$ are one such pair of Lagrange optimizers at $\mu^{*}$, and by definition they have a feasible constraint slack. Hence all Lagrange optimizers $\alpha\left(\mu^{*}\right), p\left(\mu^{*}\right)$ have the same feasible constraint slack as $\alpha^{*}, p^{*}$. Additionally all optimizers $\alpha\left(\mu^{*}\right), p\left(\mu^{*}\right)$ yield the same minimum Lagrangian value $L\left(\alpha\left(\mu^{*}\right), p\left(\mu^{*}\right), \mu^{*}\right)$. By the form of the Lagrangian in (22) it follows that all optimizers $\alpha\left(\mu^{*}\right), p\left(\mu^{*}\right)$ also give the same primal objective in (20) as the point $\alpha^{*}, p^{*}$, i.e., the minimum $P$. Hence any optimizer pair $\alpha\left(\mu^{*}\right), p\left(\mu^{*}\right)$ is primal optimal. The first statement of the theorem follows because the scheduling and power allocation obtained by (37)-(39) at $\mu^{*}$ describe one pair of Lagrange optimizers at $\mu^{*}$, i.e., are optimal solutions to Problem 1.

The convergence of iterations (33)-(34) to the optimal dual variable $\mu^{*}$ for stepsizes in (35) relies on the boundedness of the subgradient vectors (as mentioned after (32)) and follows from a standard subgradient method argument - for a proof see, e.g., [24, Prop. 8.2.6].

\section{Proof of Proposition 3}

We begin by noting that at every time $k$ the vector $s_{k}$ computed by (41) is a stochastic subgradient for the dual function $g(\mu)$ at the point $\mu_{k}$, i.e.,

$$
g\left(\mu^{\prime}\right)-g\left(\mu_{k}\right) \leq\left(\mu^{\prime}-\mu_{k}\right)^{T} \mathbb{E}\left[s_{k} \mid \mu_{k}\right] \quad \text { for all } \mu^{\prime} \in \mathbb{R}_{+}^{m} .
$$

To show this fact compare equations (40)-(41) of the online algorithm with (31) to conclude that $\mathbb{E}\left[s_{k} \mid \mu_{k}\right]=s\left(\mu_{k}\right)$ because $h_{k}$ is i.i.d for every $k$. Inequality (62) then follows directly from (30).

Then note that by Assumption 3 there exists a strictly feasible primal solution $\alpha^{\prime}, p^{\prime}$. Call $P^{\prime}$ the resulting objective value (20) at this point, and let a positive constant $\varepsilon^{\prime}>0$ denote the constraint slack of (21) at this point, i.e., $c_{i}+\varepsilon^{\prime} \leq$ $\mathbb{E}_{h} \sum_{j=1}^{f} \alpha_{i j}^{\prime}(h) q\left(h_{i j}, p_{i j}^{\prime}(h)\right)$. Then we may bound the dual function (23) at the optimal $\mu^{*}$ by

$$
\begin{aligned}
& D=g\left(\mu^{*}\right) \leq L\left(\alpha^{\prime}, p^{\prime}, \mu^{*}\right)=P^{\prime}+ \\
& \sum_{i=1}^{m} \mu_{i}^{*}\left[c_{i}-\mathbb{E}_{h} \sum_{j=1}^{f} \alpha_{i j}^{\prime}(h) q\left(h_{i j}, p_{i j}^{\prime}(h)\right)\right] \leq P^{\prime}-\sum_{i=1}^{m} \mu_{i}^{*} \varepsilon^{\prime}
\end{aligned}
$$

Rearranging the above inequality, and since $\mu^{*} \geq 0$, it follows that $\mu_{\ell}^{*} \leq \sum_{i=1}^{m} \mu_{i}^{*} \leq\left(P^{\prime}-D\right) / \varepsilon^{\prime}$ for every $\ell$, i.e., the optimal dual variables are finite.

Since the optimal dual variables are finite, the distance $\left\|\mu_{k}-\mu^{*}\right\|$ between any random $\mu_{k}$ obtained by Algorithm 1 and the set of optimal dual variables $\mu^{*}$ is a well-defined and bounded random variable. The following lemma gives an upper bound on this distance. Here recall that as we commented after (31) the subgradients $s(\mu)$ are always bounded in our problem.

Lemma 1. Let $D$ be the optimal value of the dual problem (25), $\mu^{*}$ be an optimal solution, and $S$ be the bound on the subgradient $\|s(\mu)\| \leq S$ for any $\mu \in \mathbb{R}_{+}^{m}$. Then at each step $k$ of Algorithm 1 the update of $\mu_{k+1}$ satisfies

$\mathbb{E}\left[\left\|\mu_{k+1}-\mu^{*}\right\|^{2} \mid \mu_{k}\right] \leq\left\|\mu_{k}-\mu^{*}\right\|^{2}+\varepsilon_{k}^{2} S^{2}-2 \varepsilon_{k}\left(D-g\left(\mu_{k}\right)\right)$

Proof: First use the expression $\mu_{k+1}=\left[\mu_{k}+\varepsilon_{k} s_{k}\right]_{+}$in Algorithm 1 to write

$$
\left\|\mu_{k+1}-\mu^{*}\right\|=\left\|\left[\mu_{k}+\varepsilon_{k} s_{k}\right]_{+}-\mu^{*}\right\| \leq\left\|\mu_{k}+\varepsilon_{k} s_{k}-\mu^{*}\right\|,
$$

where the last inequality holds because when projecting on the positive orthant the distance from a point $\mu^{*}$ in the orthant can only decrease. Taking expectation on both sides given $\mu_{k}$ and expanding the square norm of the right hand side, we get

$$
\begin{aligned}
\mathbb{E}\left[\left\|\mu_{k+1}-\mu^{*}\right\|^{2} \mid \mu_{k}\right] \leq & \left\|\mu_{k}-\mu^{*}\right\|^{2}+\varepsilon_{k}^{2} S^{2} \\
& +2 \varepsilon_{k}\left(\mu_{k}-\mu^{*}\right)^{T} \mathbb{E}\left[s_{k} \mid \mu_{k}\right]
\end{aligned}
$$

where we bounded $\left\|\mathbb{E}\left[s_{k} \mid \mu_{k}\right]\right\|^{2}<S^{2}$. The statement (63) follows from (65) by applying inequality (62) with the substitution $\mu^{\prime}=\mu^{*}$. 
Our goal is to use (63) to show that $\left\|\mu_{k+1}-\mu^{*}\right\|^{2} \rightarrow 0$ almost surely. To pursue this we will define a sequence that behaves as a supermartingale stochastic process and use the a.s. convergence results for such processes. In particular we will make use of the following result [27, Th. E7.4].

Theorem 3. Suppose $\left\{a_{k}, k \geq 0\right\}$ and $\left\{b_{k}, k \geq 0\right\}$ are integrable non-negative stochastic processes adapted to a filtration $\mathcal{F}_{k}$, i.e., $a_{k}, b_{k}$ measurable with respect to $\mathcal{F}_{k}$, and they also satisfy

$$
\mathbb{E}\left[a_{k+1} \mid \mathcal{F}_{k}\right] \leq a_{k}-b_{k}
$$

Then $a_{k}$ converges almost surely and $b_{k}$ is almost surely summable, i.e., $\sum_{k=0}^{\infty} b_{k}<\infty$ a.s.

To make the connection between the above theorem and (63) define

$$
\begin{aligned}
a_{k} & =\left\|\mu_{k}-\mu^{*}\right\|^{2}+\sum_{\ell=k}^{\infty} \varepsilon_{l}^{2} S^{2}, \\
b_{k} & =2 \varepsilon_{k}\left(D-g\left(\mu_{k}\right)\right),
\end{aligned}
$$

and let $\mathcal{F}_{k}=\left\{\mu_{0}, \ldots, \mu_{k}\right\}$. Note that the process $a_{k}$ is well defined because by assumption the stepsizes are square summable. Moreover $a_{k} \geq 0$ and also $b_{k} \geq 0$ because by definition $D$ is the maximum value $g\left(\mu_{k}\right)$ can take (cf. (25)). Also $a_{k}$ and $b_{k}$ are bounded variables for every $k$ because $\mu_{k}$ generated by Algorithm 1 is bounded at every $k$. Thus $a_{k}$ and $b_{k}$ are integrable, and trivially measurable with respect to $\mathcal{F}_{k}$. To check that condition (66) holds use the definition of $a_{k}$ to write

$$
\begin{aligned}
& \mathbb{E}\left[a_{k+1} \mid \mathcal{F}_{k}\right]=\mathbb{E}\left[\left\|\mu_{k+1}-\mu^{*}\right\|^{2} \mid \mu_{k}\right]+\sum_{\ell=k+1}^{\infty} \varepsilon_{l}^{2} S^{2} \\
& \leq\left\|\mu_{k}-\mu^{*}\right\|^{2}+\varepsilon_{k}^{2} S^{2}-2 \varepsilon_{k}\left(D-g\left(\mu_{k}\right)\right)+\sum_{\ell=k+1}^{\infty} \varepsilon_{l}^{2} S^{2}
\end{aligned}
$$

where for the last inequality we used (63). It is immediate that the right hand side of (69) equals $a_{k}-b_{k}$ by our appropriately constructed processes. Hence all conditions of Theorem 3 hold true.

The theorem states that $a_{k}$ converges almost surely to some random variable. Observe that the second summand $\sum_{\ell=k}^{\infty} \varepsilon_{l}^{2} S^{2}$ of $a_{k}$ in (67) is deterministic and converges to 0 because of square summability of the stepsizes. Thus we conclude that the random variable $\left\|\mu_{k}-\mu^{*}\right\|^{2}$ converges almost surely to some random variable.

To arrive at a contradiction suppose the limit random random variable is not identically zero, i.e., it takes positive values with nonzero probability. Equivalently there exist $\delta>0$ and $\varepsilon>0$ such that with probability $\delta$ we have $\left\|\mu_{k}-\mu^{*}\right\| \geq \varepsilon$ for all sufficiently large $k$. This implies that $\mu_{k}$ are bounded away from the optimal, i.e., that for sufficiently large $k$ we have $D-g\left(\mu_{k}\right) \geq \varepsilon^{\prime}$ for some $\varepsilon^{\prime}>0$. Hence with probability $\delta$ we have

$$
\sum_{k=0}^{\infty} b_{k}=\sum_{k=0}^{\infty} 2 \varepsilon_{k}\left(D-g\left(\mu_{k}\right)\right)=+\infty
$$

But this contradicts with Theorem 3 which states that $\sum_{k=0}^{\infty} b_{k}=\infty$ can only happen at a set of probability measure zero. Therefore $\left\|\mu_{k}-\mu^{*}\right\|$ must converge to zero with probability 1 .

By continuity of the concave dual function $g(\mu)$ we also have that $g\left(\mu_{k}\right)$ converge to the optimal value $g\left(\mu^{*}\right)=D$ a.s.

\section{Proof of Theorem 2}

To show that (48) holds we first convert it into an equivalent one involving variables relating to the dual problem (25). Imitating the steps leading from problem (16) to problem (20), the statement of (48) becomes equivalent to

$$
\limsup _{k \rightarrow \infty} c_{i}-\mathbb{E}_{h_{k}}\left[\sum_{j=1}^{f} \alpha_{i j, k} q\left(h_{i j, k}, p_{i j, k}\right) \mid \mu_{k}\right] \leq 0 .
$$

Here to suppress notation we have exploited the fact that according to the online algorithm the variables $\alpha_{k}, p_{k}$ depend just on the value of the variable $\mu_{k}$ and not on the whole observed channel history (but $\mu_{k}$ does depend on the whole history).

Then by the expression of $s_{k}$ given in (41) condition (71) is equivalent to $\lim \sup _{k \rightarrow \infty} \mathbb{E}_{h_{k}}\left[s_{k} \mid \mu_{k}\right] \leq 0$. Also we already argued in the proof of Prop. 3 that $\mathbb{E}_{h_{k}}\left[s_{k} \mid \mu_{k}\right]=s\left(\mu_{k}\right)$ where $s\left(\mu_{k}\right)$ is given by (31) and expresses a subgradient of the dual function $g$ at $\mu_{k}$. To sum up, we have shown so far that (48) is equivalent to $\lim \sup _{k \rightarrow \infty} s\left(\mu_{k}\right) \leq 0$.

Under Assumption 3 we have established in Proposition 3 that for the online algorithm $\mu_{k} \rightarrow \mu^{*}$ almost surely with respect to the channel sequence $\left\{h_{k}, k \geq 0\right\}$. Then we note a convex analysis fact by [24, Prop. 4.2.3]. If $g$ is concave, and $\mu_{k} \rightarrow \mu^{*}$, and $s\left(\mu_{k}\right)$ is selected as a subgradient of $g$ at $\mu_{k}$, then every limit point of $s\left(\mu_{k}\right)$ is a subgradient of $g$ at $\mu^{*}$. Hence for the sequence of $\mu_{k}$ obtained by the online algorithm we have that almost surely the sequence $s\left(\mu_{k}\right)$ converges to a subgradient of $g$ at $\mu^{*}$.

Also, as follows from Danskin's theorem [24, Prop. 4.5.1], the subgradients of the dual function $g$ at any point $\mu$ belong in the convex hull of the vectors $s(\mu)$ obtained in (31). Hence the sequence $s\left(\mu_{k}\right)$ converges almost surely to the convex hull of the vectors $s\left(\mu^{*}\right)$. But under Assumptions 1, 2, and 3, as we argued in the proof of Theorem 1 , the vectors $s\left(\mu^{*}\right)$ take a unique value that satisfies $s\left(\mu^{*}\right) \leq 0$. Hence for the sequence of $\mu_{k}$ obtained by the online algorithm we have that almost surely $\lim \sup _{k \rightarrow \infty} s\left(\mu_{k}\right) \leq 0$, which verifies statement (48).

Finally let us prove (49). Recall that the dual function equals $g(\mu)=L(\alpha(\mu), p(\mu), \mu)$ where $\alpha(\mu), p(\mu)$ are chosen as Lagrange optimizers at $\mu$ according to (24). Using the definition of the Lagrangian at (22) and the interpretation of the subgradient $s(\mu)$ at (31) as the constraint slack, we have that for any $\mu_{k}$

$$
\begin{aligned}
g\left(\mu_{k}\right) & =L\left(\alpha\left(\mu_{k}\right), p\left(\mu_{k}\right), \mu_{k}\right) \\
& =\mathbb{E}_{h} \sum_{i=1}^{m} \alpha_{i}\left(\mu_{k} ; h\right) p_{i}\left(\mu_{k} ; h\right)+\mu_{k}^{T} s\left(\mu_{k}\right)
\end{aligned}
$$


Now observe that the expectation in (49) equals the expectation given in (72) because by design of Algorithm 1 the primal variables $\alpha_{k}, p_{k}$ are selected as Lagrange optimizers at $\mu_{k}$. Therefore to show that (49) holds a.s. it suffices to show that the expectation in (72) converges a.s. to $P$ which equals $D$ by strong duality.

Proposition 3 establishes that the left hand side of (72) converges to $g\left(\mu_{k}\right) \rightarrow D$, and also that $\mu_{k} \rightarrow \mu^{*}$ a.s. We have also already argued that $s\left(\mu_{k}\right) \rightarrow s\left(\mu^{*}\right)$ a.s. Therefore also $\mu_{k}^{T} s\left(\mu_{k}\right) \rightarrow \mu^{* T} s\left(\mu^{*}\right)$ a.s. But by Prop. 1(b) $\mu^{* T} s\left(\mu^{*}\right)=0$. This shows that the expectation at the right hand side of (72) converges to $D$, which completes the proof.

\section{E. Proof of Corollary 1}

The result follows from the preceding proof of Theorem 2 . Once again imitating the steps leading from problem (16) to problem (20), we see that (50) is equivalent to

$$
c_{i}-\mathbb{E} \sum_{j=1}^{f} \alpha_{i j, k} q\left(h_{i j, k}, p_{i j, k}\right) \leq \delta^{\prime}
$$

for some appropriate positive constant $\delta^{\prime}>0$. The term on the left hand side is the expected value of the term in (71) with respect to the random sequence $\left\{h_{k}, k \geq 0\right\}$ and, as in the proof of Theorem 2, equals $\mathbb{E} s\left(\mu_{k}\right)$. Hence the result we want to prove is equivalent to $\lim _{\sup _{k \rightarrow \infty}} \mathbb{E} s\left(\mu_{k}\right) \leq 0$. In the proof of Theorem 2 we showed that the random variables $s\left(\mu_{k}\right)$ converge almost surely to $s\left(\mu^{*}\right)$, and that the limit point satisfies $s\left(\mu^{*}\right) \leq 0$. Additionally the variables $s\left(\mu_{k}\right)$ are bounded (see proof of Prop. 3), hence by the Dominated Convergence Theorem [28, Thm. 1.5.6] we conclude that $\lim _{k \rightarrow \infty} \mathbb{E} s\left(\mu_{k}\right)=\mathbb{E} \lim _{k \rightarrow \infty} s\left(\mu_{k}\right)=\mathbb{E} s\left(\mu^{*}\right) \leq 0$, where the last inequality follows from the monotonicity of expectation. This completes the proof.

\section{REFERENCES}

[1] K. Gatsis, M. Pajic, A. Ribeiro, and G. J. Pappas, "Opportunistic scheduling of control tasks over shared wireless channels," in ACM/IEEE 5th International Conference on Cyber-Physical Systems (ICCPS 2014), pp. 48-59, April 2014.

[2] J. Hespanha, P. Naghshtabrizi, and Y. Xu, "A survey of recent results in networked control systems," Proceedings of the IEEE, vol. 95, no. 1, pp. 138-162, 2007.

[3] L. Schenato, B. Sinopoli, M. Franceschetti, K. Poolla, and S. Sastry, "Foundations of control and estimation over lossy networks," Proceedings of the IEEE, vol. 95, no. 1, pp. 163-187, 2007.

[4] M. Donkers, W. Heemels, N. Van De Wouw, and L. Hetel, "Stability analysis of networked control systems using a switched linear systems approach," IEEE Transactions on Automatic Control, vol. 56, no. 9, pp. 2101-2115, 2011

[5] W. Zhang, M. Branicky, and S. Phillips, "Stability of networked control systems," IEEE Control Systems Magazine, vol. 21, no. 1, pp. 84-99, 2001.

[6] D. Hristu-Varsakelis, "Feedback control systems as users of a shared network: Communication sequences that guarantee stability," in Proc. of the 40th IEEE Conference on Decision and Control (CDC 2001), vol. 4, pp. 3631-3636, 2001.

[7] L. Zhang and D. Hristu-Varsakelis, "Communication and control codesign for networked control systems," Automatica, vol. 42, no. 6, pp. 953-958, 2006

[8] J. Le Ny, E. Feron, and G. J. Pappas, "Resource constrained lqr control under fast sampling," in Proc. of the 14th International Conference on Hybrid Systems: Computation and Control, pp. 271-280, ACM, 2011.
[9] L. Meier, J. Peschon, and R. M. Dressler, "Optimal control of measurement subsystems," IEEE Transactions on Automatic Control, vol. 12, no. 5, pp. 528-536, 1967.

[10] H. Rehbinder and M. Sanfridson, "Scheduling of a limited communication channel for optimal control," Automatica, vol. 40, no. 3, pp. 491500, 2004.

[11] G. C. Walsh, H. Ye, and L. G. Bushnell, "Stability analysis of networked control systems," IEEE Transactions on Control Systems Technology, vol. 10, no. 3, pp. 438-446, 2002.

[12] D. Hristu-Varsakelis and P. R. Kumar, "Interrupt-based feedback control over a shared communication medium," in Proc. of the 41st IEEE Conference on Decision and Control (CDC 2002), vol. 3, pp. 32233228, 2002.

[13] M. Egerstedt and Y. Wardi, "Multi-process control using queuing theory," in Proc. of the 41st IEEE Conference on Decision and Control (CDC 2002), vol. 2, pp. 1991-1996, 2002.

[14] A. Molin and S. Hirche, "Price-based adaptive scheduling in multiloop control systems with resource constraints," IEEE Transactions on Automatic Control, vol. 59, pp. 3282-3295, Dec 2014.

[15] A. Cervin and T. Henningsson, "Scheduling of event-triggered controllers on a shared network," in Proc. of the 47th IEEE Conference on Decision and Control (CDC 2008), pp. 3601-3606, 2008.

[16] M. S. Branicky, S. M. Phillips, and W. Zhang, "Scheduling and feedback co-design for networked control systems," in Proc.of the 41st IEEE Conference on Decision and Control (CDC 2002), vol. 2, pp. 12111217, 2002.

[17] J. W. Liu, Real-Time Systems. Prentice-Hall, Inc, 2000.

[18] A. Goldsmith, Wireless communications. Cambr. Univ. Press, 2005

[19] X. Liu, E. K. Chong, and N. B. Shroff, "A framework for opportunistic scheduling in wireless networks," Computer Networks, vol. 41, no. 4 pp. 451-474, 2003.

[20] L. Georgiadis, M. J. Neely, and L. Tassiulas, Resource allocation and cross-layer control in wireless networks. Now Publishers Inc, 2006.

[21] A. Ribeiro, "Optimal resource allocation in wireless communication and networking," EURASIP Journal on Wireless Communications and Networking, vol. 2012, no. 1, pp. 1-19, 2012.

[22] K. Gatsis, A. Ribeiro, and G. J. Pappas, "Optimal power management in wireless control systems," IEEE Transactions on Automatic Control, vol. 59, pp. 1495-1510, June 2014.

[23] K. Gatsis, M. Pajic, A. Ribeiro, and G. J. Pappas, "Power-aware communication for wireless sensor-actuator systems," in Proc. of the 52nd IEEE Conference on Decision and Control (CDC 2013), pp. 40064011, 2013.

[24] D. P. Bertsekas, A. Nedić, and A. E. Ozdaglar, Convex analysis and optimization. Athena Scientific, 2003.

[25] D. Bertsimas and J. N. Tsitsiklis, Introduction to linear optimization. Athena Scientific Belmont, 1997.

[26] K. Gatsis, M. Pajic, A. Ribeiro, and G. J. Pappas, "Opportunistic sensor scheduling in wireless control systems," in 53rd IEEE Conference on Decision and Control (CDC 2014), 2014. To appear.

[27] V. Solo and X. Kong, Adaptive signal processing algorithms: stability and performance. Prentice-Hall, Inc., 1994.

[28] R. Durrett, Probability: theory and examples. Cambridge University Press, 2010. 